\title{
5 Gesellschaftstheoretische Kontextualisierung
}

Das Präventionsdispositiv ist in komplexe historische und soziale Zusammenhänge eingebettet. Es entfaltet und entwickelt sich im Rahmen vielfältiger gesellschaftlicher Wandlungen und Verhältnisse und kann daher nur durch ihre theoretische Rekonstruktion überhaupt greifbar und intelligibel gemacht werden. Konkret heißt das, im Rückgriff auf Bührmann und Schneider, »[d]ie empirischen Befunde zu Kontinuität und/oder Wandel der dispositivanalytisch untersuchten sozialen Phänomene [...] in einen (gesellschafts- bzw. gegenstands-)theoretischen Deutungskontext zu stellen.«(Bührmann \& Schneider 2012: 106) Damit werden mehrere Aspekte der Dispositiventwicklung gleichzeitig gefordert.

- Zum einen wird danach gefragt, was die »historisch spezifischen Voraussetzungen für Dispositive [sind] und welche Folgen ergeben sich daraus für die (sich ändernden oder kontinuierenden) sozialen Beziehungen und Selbst-Verhältnisse von Menschen?« (a.a.O.: 105)

- Zum anderen soll »zeitdiagnostisch Auskunft über die damit verbundenen Prozesse ssozialen Wandels« (a.a.O.: 106 [Herv. i. O.]) gegeben werden, d.h. das Dispositiv in seiner räumlich-zeitlichen Entfaltung zu positionieren.

- Schließlich, bezogen auf die Anforderungen einer an Foucault anlehnenden kritischen Analyse, gilt es das Dispositiv der Prävention »im Kontext historisch konkreter Herrschafts-/Machtformationen und Wissens(an-)ordnungen $\mathrm{zu}$ analysieren.«(a.a.O.: 107)

Zusammenfassend soll also die gesamtgesellschaftliche Kontextualisierung des Präventionsdispositivs aufzeigen, wie sich die sozialen Verhältnisse und die SelbstVerhältnisse der Individuen, mithin ihre Subjektivierungsweisen, verändert und gesellschaftlich stabilisiert haben, in welchen zeitlich-räumlichen Anordnungen und sozialen Wandlungen sich das Präventionsdispositiv eingebettet und formiert hat, und welche Macht-Wissens-Komplexe mit seiner Entwicklung und Verbreitung entstanden sind.

Um auf die obengenannten Fragekomplexe antworten zu können, wird im Folgenden die Analyse der gesellschaftstheoretischen Kontextualisierung des Präventionsdispositivs anhand fünf Themenfelder - Demokratie, Arbeit, Gesundheit, Risiko, Sicherheit - bearbeitet. Die gewählten Themenfelder sollen das Untersuchen der gesellschaftlichen Kontexte und Diskurse rund um das Problem der Prävention und ihrer historischen Entwicklung und Verortung orientieren. Damit erfüllen sie die Funktion, die oft überlappenden und verschränkten Diskurse analytisch voneinander trennen zu können und dem kritischen Blick zugänglich zu machen. Außerdem wird im Anschluss an diese Themenfelder der Präventionsdispositiv in 
seinem gesellschaftlichen Wandel diskutiert, um seine intendierte wie nicht-intendierte Folgen und (Neben)-Effekte aufzuzeigen.

\subsection{Brüchige Demokratie}

Das Präventionsdispositiv kann nur unter bestimmten, und zwar liberal-demokratischen Bedingungen entstehen. Der Grund dafür ist die notwendige Freiheit, die den Individuen garantiert werden muss und durch die sie sich an dem gesellschaftlichen Leben beteiligen und damit ihre Potentiale erweitern können. In den letzten Jahrzehnten erfährt das liberal-demokratische Regierungsmodell allerdings eine mehrdimensionale Krise. Das folgende Kapitel soll daher in drei Abschnitten aufzeigen, unter welchen gesellschaftlichen Umständen das Präventionsdispositiv entstehen konnte und welche Konsequenzen das wandelnde demokratische Verständnis für seine Untersuchung hat. Konkret werden im ersten Abschnitt die Facetten der demokratischen Krise umrissen. Im zweiten Abschnitt wird dann die analytische Trennung zwischen der Politik und dem Politischen diskutiert, damit abschließend im dritten Abschnitt das Präventionsdispositiv als eine neue Regierungstechnologie dargestellt werden kann.

\subsubsection{Krise der Demokratie}

Die westlichen industriellen Gesellschaften, in denen das Präventionsdispositiv als eine immanente Regierungsform vorkommt und analysiert wird, haben ihr Demokratieverständnis seit der Antike kontinuierlich weiterentwickelt und geschärft. Die heutigen Demokratien stehen allerdings vor einer mehrdimensionalen Krise, die sich aus der "Krise des Vertrauens in die politischen Eliten, Parteien, Parlamente und Regierungen zu einer allgemeinen Krise der Demokratie« verdichtet (Merkel 2015: 7). Ihre Facetten sollen im Folgenden kurz umrissen werden:

- Eine erste Facette der demokratischen Krise liegt in dem Vertrauensverlust in die repräsentative Politik. Die Diagnose einer "partizipativen Krise der real existierenden repräsentativen Demokratie« (Saward 2010; Alonso et al. 2011 zit.n. Merkel 2015: 8) ${ }^{53}$ kann auch deshalb erstellt werden, weil die Demokratie zum einen »kein Engagement mehr « weckt (Tormey 2015: 56), und zum anderen, weil ihre Krise »auch die inoffizielle und unterirdische Politik infiziert, die Politik der Straße ebenso wie die etablierte Politik, die >Politik der Politiker «.« (ebd. [Herv. i. O.]) Der Krisenzustand partizipativer Demokratie auf beiden Ebenen lässt sich für manche Forscher auf »eine Kombination aus wachsenden

53 Hier wird die Krise im Vergleich zu zwei Referenzpunkten betrachtet. Der normative Referenzpunkt bezieht sich auf ein Ideal der Demokratie, der empirische Referenzpunkt demgegenüber auf ein vergangenes goldenes Zeitalter der Demokratie (vgl. Merkel 2015: 8). 
Erwartungen kritischer gewordener Bürger, dem Einfluss negativer Medienberichterstattung und der vor allem deshalb wahrgenommenen Performanzschwächen demokratischer Regierungen« zurückführen (Merkel 2015: 9), was demnach allerdings als ein »keineswegs existenzgefährdendes, demokratisches Defizit« (ebd.) dargestellt werden muss.

- Eine zweite Facette der Demokratiekrise besteht darin, dass es in den westlichen Stammländern der Demokratie immer offensichtlicher wird, »dass die Versprechen, die in diesem Begriff [der Demokratie, J. Z.] liegen, wohl unerfüllt bleiben werden: Die Politik verliert gegenüber der Macht der Märkte dramatisch an Boden; vermeintlich demokratische Systeme sind fest in der Hand machtvoll organisierter Interessen und haben mit Volkssouveränität - wenn es die je irgendwo gab - immer weniger zu tun.« (Blühdorn 2013: 9) Der Verlust des Gefühls, die eigenen Belange verwalten und bestimmen zu können, ohne von den transnationalen Korporationen, globalen Finanzgruppen und weiteren großen Wirtschaftskonzernen überholt zu werden, führt unausweichlich zum wachsenden Desinteresse an dem politischen Geschehen ${ }^{54}$ und, in der Konsequenz, auch zur Frustration über die Ergebnisse demokratischer Prozesse (vgl. Kersting 2008; Schäfer 2013). ${ }^{55}$

- Eine dritte Facette der Demokratiekrise besteht in dem wachsenden Abstand zwischen den Regierungen und den Bürgern und macht sich dadurch bemerkbar, dass die letztgenannten nicht in eine "politische Apathie verfallen, sondern dass sie im Gegenteil omnipräsent sind.« (Michelsen \& Walter 2013: 43) Ihre Omnipräsenz wird durch eine Art virtueller Politik gewährleistet, die durch »die einladende dialogische Geste des >Reden wir miteinander<, derzeit allgegenwärtig ist.« (ebd. [Herv. i. O.]) Somit sollen die Bürger in die aktive Gestaltung der Politik zurück miteinbezogen werden. Allerdings läuft am Ende eine solche konsultierende Partizipation »auf eine skanalisierte oder kontrollierte Politisierung hinaus, die aber insofern eigentlich entpolitisierend wirkt, als

54 So zeigt Jenni Brichzin z.B. dass »die Akzeptanz der politischen Akteure erkennbaren Schwankungen unterliegt (Schüttemeyer 1986) (Brichzin 2016: 192) und dass »das Ansehen des Politikerberufs [...] dauerhaft niedrig [ist] (Institut für Demoskopie Allensbach 2003). Zunehmend zeichnet sich Skepsis gegenüber dem politischen Prozess ab, was in den goer Jahren zu einer - von der Parteienkritik des damaligen Bundespräsidenten angestoßenen (Weizsäcker 1992) - ausgedehnten Debatte um Politikverdrossenheit führte (siehe Thierse 1993).« (Ebd.)

55 Wie der Soziologe und Zeitdiagnostiker Zygmunt Bauman bemerkt hat, bedeutet heute »der Wechsel von Regierungen - selbst von sogenannten politischen Lagern - [...] keinen Wendepunkt mehr, allenfalls ein Kräuseln an der Oberfläche eines Stromes, der dumpf entschlossen, nur von seinem eigenen inneren Impuls getrieben, unaufhaltsam und eintönig seinen Lauf nimmt.« (Bauman 2000: 11) 
dabei >Politisierung (Konflikthaftigkeit politischer Entscheidungen, die nie verschwindet) und Öffentlichkeit (im Sinne eines Aggregationsphänomens individuell-bürgerlich erfahrener Politisierung) getrennt werden und dadurch in einem gewissen, nämlich einheitsstiftenden Sinne entschärft werden` (Teßmer 2012: 173) können.« (Michelsen \& Walter 2013: 44 [Herv. i. O.])

- Eine vierte Facette des Krisenzustands ließe sich an Einzelerscheinungen beschreiben, die solche Entwicklungen beinhalten wie z.B. »die `Oligarchisierung der liberalen Demokratie (Buchstein), die Aushöhlung der Gewaltenteilung durch >Präsidentialisierung ‘ (Körösenyi), die Entstehung des >Populismus statt demokratischer Partizipation, und die >Dominanz korporatistischer Arrangements` im Entscheidungsprozess (Colin Crouch).« (von Beyme 2013: 12 [Herv. i. O.] $)^{56}$ All diese Einzelerscheinungen fassen die Schwierigkeiten, vor denen die heutigen demokratischen Ordnungen stehen, zusammen. Sie lassen sich in unterschiedlichen Ausprägungen vorfinden und beschreiben funktionale Defizite der Demokratie, die aber, wenn sie zusammen und in kürzeren $\mathrm{Ab}$ ständen erscheinen, zu bedrohenden Entwicklungen führen können, was der Populismuszuwachs wohl am besten bestätigt.

Die vier knapp behandelten Facetten weisen auf das Erleben einer Fassade formeller Prinzipien hin, was die Forscher mit einer Entfernung von der idealen Demokratie identifizieren und mit dem Namen Post-Demokratie bzw. Post-Politik bezeichnen (vgl. Dahrendorf 2001; Crouch [2003] 2008; Fach 2008; Serloth 2009; Konicz 2012; Förster \& Lemke 2017). Mit einer solchen Bezeichnung sollte zum Ausdruck gebracht werden, dass

»moderne, westliche Demokratien hinter einer Fassade formeller demokratischer Prinzipien und unterstützt durch das Hegemonialwerden neoliberaler Denkweisen zunehmend von Eliten kontrolliert werden. An die Stelle der Selbstregierung des Volkes sei ein politisches System getreten, das maßgeblich via Massenmedien durch die Interessen von Unternehmen, Verbänden und gesellschaftlichen Eliten gesteuert werde, während die Bürger in zum >Spektakek (Crouch 2008a: 10) verkommenen Wahlen nur noch scheinbar die Geschicke ihres Gemeinwesens bestimmen, de facto aber nicht mehr zwischen klaren und durch sie steuerbaren politischen Alternativen wählen können.«(Ritzi 2014: 2 [Herv. i. O.])

56 Auch die demokratischen Defizite der Europäischen Union, wie sie der britische Politikwissenschaftler Simon Hix zusammen mit Andreas Follesdal herausgearbeitet haben, verweisen auf den allgemein verstärkten Kontrast zwischen den vorgeschriebenen, de jure existierenden demokratischen Prozessen und der tatsächlichen, de facto erlebten Demokratie (vgl. Follesdal \& Hix 2006: 534ff). 
Das damit begonnene Zeitalter der Post-Demokratie, der Post-Politik, aber auch des Post-Fundaments (vgl. Marchart 2010) und neuerdings auch des PostFaktums ${ }^{57}$, eröffnet neue Perspektiven auf Demokratie und Politik. Bereits Ende des Jahres 1968 resümierte Gräfin Dönhoff, Chefredakteurin und Mitherausgeberin der deutschen Wochenzeitung Die Zeit, die Diskussion um die Krise der Demokratie folgenderweise: »Seit Wochen wurde hier an dieser Stelle die Frage abgehandelt: Ist die parlamentarische Demokratie noch fähig, mit den Konflikten der heutigen differenzierten Gesellschaft fertig zu werden? Ist sie noch in der Lage, ihre Aufgabe wahrzunehmen: politische Entscheidungen zu fällen und Kontrollen auszuüben?« (Dönhoff 1968) Die Antworten auf diese grundlegenden Fragen wurden in dem letzten Jahrhundert von zwei dominierenden politischen Ordnungen, der demokratisch-liberalen und der real-sozialistischen Regierungsordnung, gesucht. Doch seit dem Fall des sozialistischen Systems am Ende des letzten Jahrhunderts (vgl. Ritzi 2014: 11) und dem Zusammenbruch der liberal-demokratischen politischen Utopie am Anfang des neuen Jahrhunderts (vgl. Žižek 2009) vermehren sich die Krisendiagnosen der Demokratie exponentiell. Dass die Demokratie eine erneute Reflexion benötigt, zeigen auch die zahlreichen gegenwärtigen Versuche ihrer Auslegung und Neubestimmung: »Wie ist die Politik zu denken, wenn sie weder in dem, was die westlichen Demokratien unter Politik verstehen, noch in den realsozialistischen Vorstellungen - und was von ihnen übrig blieb - aufgeht?« (Hebekus \& Völker 2012: 12) ${ }^{58}$ Um die Wurzeln dieser Entwicklung und der damit einhergehenden Problematisierung des Regierens verfolgen $\mathrm{zu}$ können, wird es zunächst notwendig, eine begriffliche Unterscheidung zwischen der Politik und dem Politischen vorzunehmen.

\subsubsection{Die Politik und das Politische}

Die Geschichte der Differenzierung zwischen der Politik und dem, was das Politische genannt wird, geht auf Paul Ricœur zurück, welcher in der Reaktion auf die

57 Postfaktisch ist das internationale Wort des Jahres 2016. Es besagt, dass nicht mehr die Tatsachen (Fakten) über der Wahrheit einer Aussage entscheiden, sondern die dahinterstehenden emotionalen Reaktionen (Affekte). Den hiermit verbundenen Zuwachs von populistischen Stimmungen hat schon vor fünfzehn Jahren Ralf Dahrendorf auf die mangelnde demokratische Auseinandersetzung zurückgeführt, die eine informierte und besonnene Debatte über große Fragen ermöglichen sollte: »In der traditionellen Demokratie lag hier die Aufgabe der Parlamente. Je schwächer aber die Parlamente werden und je mehr sie diese Rolle verlieren, umso weniger Chancen bestehen für eine solche demokratische Debatte und desto mehr ungerechtfertigte Macht eignen sich die neuen Vermittler an. Populisten treiben diesen Prozess bewusst voran mit dem Ziel, die Debatte zu überspringen und einen Konsens auf Crund von vermeintlichen oder tatsächlichen, mehr oder weniger tief empfundenen Gefühlen der Bevölkerung zu schaffen. « (Dahrendorf [2001] 2002: 89f)

58 Vgl. dazu auch weitere Abhandlungen: Rancière 2012; Wilson \& Swyngedouw 2014; Dubreuil [2012] 2016 
Besetzung Ungarns 1956 die doppelte Originalität des Politischen herauszuarbeiten versuchte.

Die Autonomie des Politischen (franz. du politique) gründet seiner Meinung nach in zwei gegensätzlichen Zügen: »Auf der einen Seite stellt das Politische (le politique) ein menschliches Verhältnis her, das sich nicht auf die sozio-ökonomischen Spannungen der Gesellschaft reduzieren lässt [...]. Auf der anderen Seite bringt die Politik (la politique) spezifische >Übel hervor, politische Übel eben, Übel der politischen Macht." (Ricœur 1974: 249 zit.n. Marchart 2010: 32 [Herv. i. O.]) An dieser Stelle gilt es zwischen drei Begriffen zu unterscheiden. Ricœur weist dem Bereich des Politischen ( $d u$ politique) eine spezifische Autonomie $\mathrm{zu}$, welche neben den anderen Dimensionen des gesellschaftlichen Lebens verortet ist (der Ökonomie, dem Sozialen, dem Kulturellen u.a.) (vgl. Nissen \& Vobruba 2009; Faßler 2014; Bonz 2011). Innerhalb dieser Sphäre unterscheidet er dann zwischen zwei ihrer Wesenszüge. Zum einen gründet die Autonomie des Politischen ( $d u$ politique) in dem Politischen (le politique), welches die grundsätzlichen Verhältnisse des Zusammenlebens umfasst, die außerhalb jeglicher Konfliktlinien fungieren. Zum anderen gründet diese Autonomie des Politischen (du politique) in der Politik (la politique), in welcher die Machtgier und die der Gesellschaft unterliegenden Spannungen und Konflikte zum Ausdruck kommen. Aus diesen zwei Wesenszügen besteht laut Ricœur die Autonomie des Politischen (franz. autonomie du politique), welche »durch das doppelte Merkmal >des Politischen « und >der Politik bestimmt [ist], d.h. durch eine ideale Sphäre des Politischen (des rationale Übereinstimmung verkörpernden Gemeinwesens im Sinne des englischen polity), die durch eine spezifische Rationalität definiert wird, und eine Sphäre der Macht oder Politik." (Marchart 2010: 33 [Herv. i. O.]) Es ist diese paradoxe Spannung, die dem Begriff des Politischen (du politique) bei Ricœur innewohnend ist. Ihr Kern besteht in der Überlagerung "von Idealität und Realität, von polity und policy, von Vernunft und Macht.« (Dallmayr 1993b: 187 zit.n. Marchart 2010: 34 [Herv. i. O.]) Eine tiefgehende und breiter angelegte Diskussion um den Inhalt und die Bedeutung des Politischen fängt jedoch erst mit der Gründung des Zentrums für philosophische Studien über das Politische (franz. Centre de recherches philosophiques sur le politique) bei Philippe Lacoue-Labarthe und Jean-Luc Nancy im Jahr 1980 statt.

Lacoue-Labarthe und Nancy haben den Begriff des Politischen auf doppelte Weise hinterfragt. Zum einen ging es den beiden darum, das Politische in seiner philosophischen Qualität zu untersuchen. Das Politische steht demnach in einer engen Beziehung zu dem Philosophischen. Es war also notwendig, im Unterschied zu Ricœur, der das Politische neben anderen Dimensionen des gesellschaftlichen Lebens verortete, das Politische, seine Begründung und seine Essenz aus der Sicht der Philosophie her zu hinterfragen. Aus dieser Sicht zielt dann das Politische »auf die Ebene der grundsätzlichen Konfiguration gemeinschaftlicher Zusammenhänge [...], so wie sie etwa in vielen Gründungserzählungen erfasst werden.« (Hebe- 
kus \& Völker 2012: 15) Zum anderen war es für beide Philosophen unabdingbar, zu der »eigentlich politischen Voraussetzung der Philosophie (bzw. wenn man es vorzieht: der Metaphysik), das heißt bis zu einer politischen Bestimmung der Essenz (Lacoue-Labarthe \& Nancy, 1981: 15) [zurückzukehren].« (Ebd.) Für Nancy und Lacoue-Labarthe erscheinen also das Politische und das Philosophische in einem engen Zusammenhang. Das Politische gilt es aus diesem Grund zuerst von seinen analytischen, empirischen oder hermeneutischen Bedeutungen abzugrenzen und es in seiner grundlegenden philosophischen Fragestellung erscheinen zu lassen. Das Philosophische ist aber wiederum durch seine ursprüngliche politische Dimension bestimmt, und zwar der Frage nach dem ersten Grund des Gemeinwesens, d.h. nach der Essenz des Politischen:

»Es geht also nicht um die eine oder andere politische Verankerung der ein oder anderen Idee des Politischen, sondern es geht um die grundsätzliche Bestimmung der Essenz des Politischen als philosophischer bzw. noch umfassender um die politisch-philosophische Einsetzung des abendländischen Denkens und die Praxis der Gemeinschaft. Um diese intrinsische Verknüpfung zu bezeichnen, sprechen die Autoren im Unterschied zur Rede von >dem< Politischen von der >Essenz $<$ des Politischen, deren Grund im Denken der antiken polis liegt, in welchem das okzidentale Verständnis der Philosophie und des Politischen entsteht. Zu dieser doppelten Eröffnung ist also zurückzukehren, will man die gegenwärtige Situation verstehen, die durch den >Entzug des Politischen ` geprägt ist. « (Hebekus \& Völker 2012: 16 [Herv. i. O.])

Wie angedeutet, hinterfragen Nancy und Lacoue-Labarthe das Politische in seiner Bedeutung tiefer und erörtern die wechselseitige Bestimmung des Politischen und des Philosophischen, die bereits in der antiken philosophischen Erfahrung des Gemeinschaftslebens verwurzelt ist. Geprägt von ihrem Mitarbeiter Jean-François Lyotard, welcher symbolisch das Ende der großen Erzählungen ausgerufen hat ${ }^{59}$, erweist sich dieser methodologische Vorgang als unabdingbar für die weitere Reflexion des Politischen und der Demokratie. Die großen Diskurse der Geschichte, die Meta-Erzählungen, sind nämlich seiner Meinung nach an ihr Ende gekommen. Es gibt keine allgemein gültige Wahrheit mehr, kein letztes Ziel der Geschichte, auf

59 Jean-François Lyotard zielte hiermit auf die Tatsache, dass sich »die von der Aufklärung bzw. der Moderne verkündete >Croße Erzählung vom Fortschritt der Menschheit, der Vernunft oder der Harmonie einer klassenlosen Cesellschaft [...] als Trugbild eines heilsgeschichtlichen Versprechens erweist. Das Projekt der Moderne jedoch, so Lyotards Befund, ist nicht abgeschlossen, muss aber die von ihr unbemerkten narrativen Muster des Fortschritts bzw. des erfüllbaren Sinns von Ceschichte als Fiktion, als `Metaerzählung، reflektieren.« (Tholen 2005: 308 [Herv. i. O.]) Dadurch wollte Lyotard nicht zuletzt auf die Delegitimierung der politischen Weltanschauungen und der metaphysischen Cründungsgeste der Philosophie hinweisen (vgl. Bedorf \& Röttgers 2010: 14). 
den hin sich die Politik ausrichten könnte (vgl. Lacoue-Labarthe \& Nancy [1982] 1997: 130f). Der Verlust des Glaubens an große Erzählungen, an einigende Ideen und Werte nötigt deshalb dazu, »die Einrichtung der Gesellschaft als unendlich politische Aufgabe zu begreifen, als unüberschaubares Ensemble von Konfliktlinien, an denen immer wieder neue Entscheidungen zu treffen sind.« (Flügel et al. 2004: 11) Das Politische gilt es deshalb aus ihrer Sicht neu zu begründen und ihm eine neue Bedeutung zuzuweisen.

Zudem sprechen beide Autoren von dem Entzug des Politischen. Damit meinen sie, dass das hier beschriebene Ende der großen Erzählungen nicht nur zu einem Ende führt, sondern dass es sich um den Prozess einer Erfüllung und eines Endes handelt, »der zu einer Despezifizierung des Politischen führt, zu seiner Verallgemeinerung, so dass sich das Politische schließlich zugleich als omnipräsent und verschwunden bzw. entleert erweist. (Hebekus \& Völker 2012: 16) Jeder Ort des Politischen wird verloren, »es taucht überall auf und nirgends. "(Flügel et al. 2004: 11) In einem solchen Prozess erfüllt sich und entzieht sich das Politische zur gleichen Zeit. Dieser doppelten Bewegung der Erfüllung und des Endes liegt die Annahme zugrunde, dass der gegenwärtige Mensch, mit Hannah Arendt argumentierend, als Schöpfer seiner selbst (lat. animal laborans) in einem sozialen, von politischer Qualität gelösten - sprich immanenten - Raum lebt, in dem die Unterscheidung zwischen der Freiheit und der Autorität schwindet (vgl. Nancy \& Lacoue-Labarthe 1983: 192 zit.n. Hebekus \& Völker 2012: 18).

Das Schwindung der Differenz zwischen Freiheit und Autorität wird durch die Auflösung der Transzendenz charakterisiert. Im Zeitalter des klassischen Totalitarismus legitimierte nämlich die Transzendenz bzw. die Alterität (Andersartigkeit) des Regierenden seine Macht. Lacoue-Labarthe und Nancy skizzieren in dieser Hinsicht drei Ursprünge einer solchen Alterität: sie könnte entweder als eine gleichzeitige Artikulation der Macht als einer Gewalt und der Autorität als einer Transzendenz entstehen (Faschismus), oder ihren Ursprung in der Beziehung der Gemeinschaft zu einer unvergänglichen Idee haben (Kommunismus), oder aber sich als eine Beziehung der Gemeinschaft zu sich selbst entwickeln (Selbstdarstellung des italienischen Faschismus unter Mussolini) (vgl. Lacoue-Labarthe \& Nancy [1982] 1997: 130). Anstelle der Inkorporation der Transzendenz im klassischen Totalitarismus erleben wir aber, gerade durch die Streichung der Differenz zwischen Freiheit und Autorität, eine Auflösung der Transzendenz. Beide Autoren begreifen deswegen den Entzug des Politischen als den Entzug der Transzendenz (vgl. a.a.O.: 129). Die Folgen dieses Entzugs zeigen sich dann in dem Einzug eines neuen, weichen Totalitarismus, der alle Sphären des menschlichen Lebens umfängt. ${ }^{60}$ Dieser

60 Das Wortspiel Entzug - Einzug ist in dem verwendeten französischen Begriff retrait angelegt. Dieser Ausdruck soll auch auf die Möglichkeit eines Neuanfangs hinweisen: »Das Verb retracer, auf das der re-trait zurückgeht, bedeutet eben auch, die Konturen neu zu ziehen.« 
Totalitarismus entsteht entlang einer neuen Homogenisierung des Politischen und wird über diskursive Techniken und eine Ideologie des Konsenses erschaffen (vgl. Hebekus \& Völker 2012: 22), was im Verweis auf die grundlegende Konflikthaftigkeit der Gesellschaft kritisiert wird (vgl. Mouffe 2007; 2014).

Als Schlussfolgerung dieses Entzugs und des Einzugs einer neuen, weicheren Spielart von Totalitarismus wird die Tatsache genannt, dass Demokratie »nicht mehr als Gegensatz des Totalitarismus verstanden werden [kann], sondern die Ununterscheidbarkeit beider [...] ein neues, kritisches Nachdenken über den Begriff der Demokratie selbst [erfordert].«(Ebd.) Und es ist die Herausarbeitung der Frage nach dem Entzug des Politischen, die dann zu der Formulierung der Essenz des Politischen beitragen soll (vgl. a.a.O.: 24). ${ }^{61}$ In Abgrenzung zu den, heute wie damals aktuellen Formen der Kritik, vor allem der Dekonstruktion, sehen LacoueLabarthe und Nancy die Aufgabe des kritischen philosophischen Zugangs jedoch darin, eine Haltung einzunehmen, die »die Bedingungen der Unmöglichkeit als aktuelle Bedingungen der Möglichkeit« (a.a.O.: 23) versteht. Das heißt, dass sie in der Unmöglichkeit einer endgültigen Definition des Politischen, welche auf ein eigenständiges Prinzip oder auf ein Axiom zurückgeführt werden müsste, die Möglichkeit und sogar die Notwendigkeit sieht, das Politische neu zu denken und neu zu erfinden (vgl. Lacoue-Labarthe \& Nancy [1982] 1997: 131). ${ }^{62}$ Die Art und Weise wie uns das Politische und seine Essenz oder, um es mit Worten von Lacoue-Labarthe und Nancy wiederzugeben, wie uns das Konzept der politischen Transzendenz dazu verpflichtet, es erneut aufzunehmen und zu durchdenken (vgl. a.a.O.: 129f), wird

(Bedorf \& Röttgers 2010: 14) Deshalb kommt neben der ersten (rückziehen) und der zweiten (entziehen) auch eine dritte (noch einmal ziehen) Bedeutung dem Begriff zu (vgl. Hebekus \& Völker 2012: 17).

61 Dabei gilt es auf das Konzept der Negativität zu achten und dieses herauszuarbeiten, denn in der Kritik des neuen Begriffs des Politischen bzw. seiner Essenz besteht die Aufgabe erstens darin, eine notwendige kritische Distanz zu dem Politischen einzunehmen, und zweitens darin, den reduzierenden Dualismus zwischen dem Realsozialismus und dem Kapitalismus zu überwinden (vgl. Hebekus \& Völker 2012: 24).

62 Im Vergleich zu Lacoue-Labarthe und Nancy meint bspw. André Brodocz, dass wir für die Unentscheidbarkeiten, »die wir nicht nicht-entscheiden können, [...] einen neuen, einen `dynamischen Begriff des Politischen als kontinuierlichen Kampf um Kontinuierung»«brauchen (Brodocz 2007: 171 [Herv. i. O.]) Er schlägt dabei vor, zwischen einer symbolischen Dimension, welche die Macht fokussiert, die die Konstitution sozialer Einheiten durchzieht und die als soziale Praxis immer wieder neu hergestellt werden muss, und einer instrumentellen Dimension, welche sich auf die Macht bezieht, die bei der Strukturierung sozialer Einheiten zum Einsatz kommt, welche wiederum durch die Handlungen der Akteure immer wieder neu hervorgebracht werden müssen, zu unterscheiden (vgl. ebd.). Für Oliver Marchart demgegenüber muss sich das politische Denken als Denken des Politischen neu erfinden, und zwar deshalb, »weil das Politische auf die Frage der Cründung verweist, die sich jeder Cesellschaft stellt, sobald sich die Gewissheiten, Prinzipien und Werte, auf denen sie gebaut ist, als fungibel erwiesen haben.« (Marchart 2010: 8) 
auch darüber entscheiden, welche kritisch-theoretische Fragestellungen sich daraus in Bezug auf die Demokratie und die ihr innewohnende Untrennbarkeit von den Formen des Totalitarismus, zu dessen weichen Formen das Präventionsdispositiv gehört, entwickeln lassen.

Zusammenfassend lassen sich folgende Aspekte der Differenzierung zwischen der Politik und dem Politischen benennen:

- Erstens gilt es die Begriffe der Politik und des Politischen klar voneinander zu trennen. Während die Politik einen Bereich der Praxis (Ricœurs la politique) und ein konkretes Verfahren auf der ontischen Ebene beschreibt, wird mit dem Begriff des Politischen auf philosophische Bedingtheit (Nancys und LacoueLabarthes Essenz) und auf die rationale gemeinschaftliche Übereinstimmung auf der ontologischen Ebene hingewiesen.

- Zweitens befinden sich die Wurzeln der Krise in der doppelten Bewegung des Entzugs und des Einzugs des Politischen, da jede Erfüllung gemeinschaftlicher Ziele zu einer Auflösung, einem Entzug des Politischen führt, wodurch sich das Politische zur Neubestimmung bereitstellt. Deshalb bedarf es einer ständigen Überprüfung der Formen des Regierens, um die darin enthaltenen Totalisierungsprozesse dechiffrieren zu können.

- Drittens, die Totalisierungsprozesse entstehen durch das Schwinden der Differenz zwischen Freiheit und Autorität und der damit einhergehenden Immanent-Werdung des sozialen Raumes. Die Auflösung der Transzendenz in eine weichere Form von Totalitarismus erfordert demnach eine kritische Untersuchung der bestehenden immanenten Regierungsordnungen und -technologien, wie denen des Präventionsdispositivs.

Auf der ontologischen Ebene lässt sich daher die Krise der Demokratie vor dem Hintergrund dessen betrachten, wie der Begriff des Politischen bestimmt und ausgelegt wird. Wenn das Politische als Inbegriff des Regierens verstanden wird, gilt es seiner eigentlichen Essenz eine inhärente Spannung zuzuweisen. Oliver Marchart spricht in diesem Sinne von dem assoziativen (auf das Zusammenleben gerichteten) und von dem dissoziativen (auf die Machtausübung gerichteten) Aspekt des Politischen. Aus der Konzentration auf den einen oder den anderen Aspekt lassen sich dann zwei Traditionslinien bzw. Forschungsperspektiven beschreiben:

- Zum einen ließe sich die sog. arendtianische Traditionslinie benennen, die auf den assoziativen Aspekt des Politischen fokussiert, die Bedeutung des Raums der Freiheit und der Deliberation betont und auf die Möglichkeiten und Hindernisse des gesellschaftlichen Zusammenlebens sowie seiner vernünftigen und ideellen Einrichtung und Regierung hinweist. 
- Zum anderen wäre die sog. schmittianische Traditionslinie zu nennen. Diese richtet ihren Blick auf den dissoziativen Aspekt des Politischen und hebt die Wirksamkeit von Machtverhältnissen hervor. Dabei untersucht sie die Konfliktlinien, Brüche, Spaltungen und Antagonismen, die aus und in den Versuchen, eine Regierungsordnung herzustellen, entstehen (vgl. Marchart 2010: 35ff).

Der zweitgenannten Tradition wird die Analyse der Regierungstechnologien zugeordnet, die in der Gesellschaft immanente Machteffekte zeitigen und zu einer weichen Totalisierung des sozialen Raumes führen. Die Kritik dieser Regierungstechnologien besteht darin, ihre Mechanismen, zugrundeliegenden Rationalitäten und herrschenden Diskurse zu durchleuchten und zu hinterfragen.

\subsubsection{Präventionsdispositiv als Regierungstechnologie}

Wird der Präventionsdispositiv als Regierungstechnologie bezeichnet und auf seine Machteffekte hinterfragt, die nicht als »ursprüngliche Gegebenheiten« (Kerchner 2006: 151), sondern als auf Handlung anderer gerichtete Handlungen (vgl. Foucault [1982] 2005: 285) gedacht werden, so geschieht dies vor dem Hintergrund des Machtbegriffes, mit dem Michel Foucault gearbeitet hat. Diese Handlungen operieren in einem gesellschaftlichen Kontext und schaffen bestimmte Dispositionen, d.h. Räume, Rationalitäten und Mittel, die es ermöglichen, andere Menschen zu gewissen Handlungen zu führen. In diesem Sinne stellt ein Dispositiv der Prävention solche Räume, Rationalitäten und Mittel - wie z.B. Subjektivierungsweisen dar, die es möglich machen, die Menschen zum präventiven Umgang mit sich selbst $\mathrm{zu}$ führen. Dieser Mechanismus bleibt insofern problematisch, als es vorbeugende Maßnahmen als Mittel zum Erfolg stilisiert, deren Gegenpol die Niederlage in Form von Erschöpfung, Depression oder Burnout ist. So fixiert er in den gesellschaftlichen Diskursen die Vorstellung, dass hohe Leistungen gepaart mit präventivem Umgang soziale Sicherheit und Anerkennung sichern können, wogegen der Verzicht auf Prävention oder die Wahl einer anderen Lebensweise diese mindert. Belohnt wird daher nicht nur die Leistung, sondern die Leistung mit Prävention, und damit die anscheinend klügere Alternative, die der Gesellschaft vorteilhaft ist und gefördert werden soll. Damit funktionalisiert sich der Umgang mit sich selbst, der einerseits dem ökonomischen Zweck der Selbstmaximierung dient und andererseits ein garantiertes Handlungsmanual zur Sicherung der eigenen Gesundheit enthält.

Der funktionalisierte Umgang mit sich selbst ließe sich mit der anfangs angedeuteten Krise der Demokratie und der Problematisierung des Politischen als zwei Seiten einer Medaille betrachten. Wird einerseits die Demokratie von der Perspektive eines Denkens des Politischen »als ein kritisch-subversiver Modus des Befragens begriffen« (Flügel-Martinsen 2017: 239), welcher »von Versuchen der Be- 
gründung eines festen Modells der Demokratie absieht « (ebd.), so wird andererseits durch das Wirken des Präventionsdispositivs ein funktionaler Umgang mit sich selbst befördert, welcher in immer intimere Bereiche der Subjekte eindringt und die politische Macht an ihre Körper - und Psyche - festmacht (vgl. Foucault [1973] 2015: 91f). Der Kampf um die Demokratie als »eine Selbstregierungspraxis jenseits letzter Gewissheiten « (Flügel-Martinsen 2017: 240) verbindet sich hier mit der Kritik an einer Machtform, »durch die das Individuum dazu gelangt, sich selbst als Subjekt zu konstituieren." (Foucault [1983] 2009: 18) Damit soll das Präventionsdispositiv jenen Techniken der Macht zugeordnet werden, mit deren Hilfe die gegenseitige und wechselhafte »Ko-Formierung von modernem souveränen Staat und modernem autonomen Subjekt « (vgl. Lemke 2000: 32f) stattfindet, was seine politikwissenschaftliche Erforschung und Kritik erzwingt.

\subsection{Wandelnde Arbeitsverhältnisse}

Arbeitsverhältnisse konstituieren einen Bereich, in dem das Wirken des Präventionsdispositivs ganz konkret beobachtet und festgestellt werden kann. Die Untersuchung der wandelnden Arbeitsbeziehungen und der damit einhergehenden Anforderungen an die Individuen können daher nahelegen, wie der Wirkungsmechanismus in der Tat funktioniert. Darum wird im Folgenden zuerst der neoliberale Wandel und die Umdeutung von Arbeits- und Regierungsverhältnissen diskutiert. Im Anschluss daran werden die aus unterschiedlichen Stellen an die Subjekte gestellten Anforderungen untersucht, sowie ihre dazu notwendigen Mittel gezeigt. Im abschließenden Teil werden die Effekte einer doppelten Selbstregierung in den Arbeitsverhältnissen problematisiert und ihr Bezug zum Präventionsdispositiv skizziert.

\subsubsection{Neoliberale Umdeutung von Arbeits- und Regierungsverhältnissen}

Arbeitsverhältnisse werden als eine Sphäre bezeichnet, wo psychisches Leiden am deutlichsten zum Ausdruck kommt (vgl. Iacovides et al. 2003; Tröster 2013; Bakker \& Costa 2014; Bianchi et al. 2014). Wenn die Individuen nicht mehr die erwünschte Leistung erbringen können, zeigt sich dies deutlich an wachsenden Fehltagen und der damit einhergehenden sinkenden Produktion bzw. Wettbewerbsfähigkeit des Unternehmens. Dadurch wird nicht nur die wirtschaftliche Seite des Problems der Gesundheit von Arbeitnehmern thematisiert, sondern ein generelles Unbehagen angesichts des psychischen Wohlbefindens, wie dies Patrick Kury in seiner Studie zur Geschichte des Stresses in den westlichen Gesellschaften rekonstruiert hat (vgl. Kury 2012). Damit bieten die wandelnden Arbeitsformen eine passende Negativfolie, die die gesellschaftliche Problematisierung der psychischen Gesundheit veranschaulichen kann. 
Aktuell wird die oben angedeutete Wandlung durch die neoliberale Ausprägung wirtschaftlicher und gesellschaftlicher Prozesse gekennzeichnet. Neoliberalismus als ökonomisches Denk- und Handlungsmuster grenzt sich von der liberalen Vorstellung vom Individuum $a b$, das sich zielgerichtet nach seiner ProfitMaximierung sehnt, rational kalkuliert und seinen Nutzen bei allen seinen Entscheidungen sucht. Stattdessen wird das moderne Individuum im Neoliberalismus vom Wirtschafts-objekt, d.h. einer auf dem freien Markt verhandelbaren Arbeitskraft, zum Wirtschafts-subjekt, d.h. einem aktiven Teilnehmer der Wirtschaftsverhältnisse umdefiniert. Ein solches Wirtschaftssubjekt muss sich selbst regulieren, aus den verschiedenen Arbeitsmarktoptionen die aktuellsten, profitabelsten und innovativsten wählen, damit es sich in dem Wettbewerb mit anderen Individuen und ähnlichen Selbstunternehmern durchsetzen kann.

Die Individuen als Wirtschaftssubjekte leben heute in Arbeitsverhältnissen, die sich durch wachsende Flexibilisierung und Prekarisierung von Arbeit, Entgrenzung zwischen Privat- und Arbeitszeit (vgl. Flick 2013: 19), ständige Erreichbarkeit, Abgrenzungsschwierigkeiten (vgl. Voswinkel 2017: 76) und immer neue Ansprüche an Produktivität, Effizienz, Kreativität und Innovationskraft (vgl. Boltanski \& Chiapello 2010: 20ff) auszeichnen. Wenn sie dann an den immer höheren Anforderungen scheitern und nicht mehr mithalten können - was in Form von Arbeitslosigkeit, Armut oder psychischem Leiden wie Burnout-Syndrom gedeutet wird - , richtet sich die Aufmerksamkeit auf ihre individuellen Eigenschaften und Kompetenzen, die gestärkt oder erweitert werden müssen, damit sie weiter konkurrenz- und produktionsfähig bleiben. Gegen diese Vorstellung eines Individuums als Selbstbzw. Arbeitskraftunternehmers (vgl. Bröckling 2007; Pongratz \& Voß 2003) richten sich zahlreiche kritische Stimmen, die die wandelnden Arbeitsprozesse und die damit entstehenden Subjektivierungsformen als Gegenstand einer neuen, neoliberalen Regierungspraxis betrachten, welche im direkten Verhältnis zum Dispositiv der Prävention steht.

Die neoliberale Denk- und Handlungsrationalität regiert durch die Anrufung und Zurichtung eines Subjektmodus, eines Homo oeconomicus. Wurde im Liberalismus des 18. und 19. Jahrhunderts das Individuum als rational handelndes Wesen anthropologisch vorausgesetzt, so wird im Neoliberalismus des 20. und 21. Jahrhunderts der Homo oeconomicus als ein künstlich formbares Wesen betrachtet, das zwar den wirtschaftlichen Profit sucht, doch die Dynamik der Marktprozesse nicht überblicken kann. Die sodann suggerierte Omnipräsenz des Marktes ermöglicht es, die Individuen stets als ökonomisch Handelnde anzurufen und sie durch Bereitstellung von Freiheiten zur permanenten Entscheidungsfindung anzuleiten (vgl. Bröckling 2004: 63). Diese Entscheidungsfindung bzw. Selbstregierung erschien im Verlauf der Zeit als eine effizientere Regierungsweise im Vergleich zum direkten Zwang und körperlicher Gewalt. Um die Menschen auf einem abgegrenzten Territorium zu regieren und regierbar zu machen, bedarf es nur indirekter Ein- 
flussnahme um »prophylaktisch den Marktprozess sicherzustellen.« (Mönch 2018: 247) Somit ließe sich das liberale und neoliberale Regieren nicht nur als eine ökonomische Schule, Wirtschaftstheorie oder gar Ideologie lesen, sondern auch, in Anlehnung an Michel Foucault, »als eine Praxis, das heißt als eine auf Ziele hin orientierte und sich durch kontinuierliche Reflexion regulierende >Weise des Tuns «." (Foucault [1979] 2003: 1020f [Herv. i. O.]) Diese Weise des Tuns braucht selbst-regulierende und also selbst-regierende Subjekte, die genau mit der Figur des Homo oeconomicus übereinstimmen. Damit wurde eine Schnittstelle zwischen Neoliberalismus und Regierungspraxis geschaffen, die es ermöglicht, die bei den Arbeitsprozessen stattfindenden Wandlungen gleichzeitig als Regierungs- und Machteffekte zu deuten (vgl. Mönch 2018: 257f).

\subsection{2 Äußere und verinnerlichte Ansprüche des neoliberalen Subjekts}

Wie im vorigen Abschnitt gezeigt, hat in den letzten Jahrzehnten neben dem Strukturwandel der Arbeitsverhältnisse auch ein Wandel der Subjektformen stattgefunden. Im Zentrum der neoliberalen Ansprüche an die Individuen steht ein Appell an die Meisterung einer modernen Optimierungs- und Leistungskunst. Leistung als solche wird zum wichtigsten Prinzip und »Maßstab für die Bewertung der eigenen Arbeit« (Kratzer et al. 2015: 164) und zu einem gesellschaftlichen Anerkennungsmerkmal erhoben. Diese Ansprüche werden von den Individuen weder erzwungen noch erwünscht. Sie erscheinen als bloße Gegebenheiten, denen sie sich intuitiv anpassen und die für sie "als objektive, interessenneutrale >Sachnotwendigkeiten in Erscheinung treten.« (Böhle 2003: 140 zit.n. Mönch 2018: 276 [Herv. i. O.]) Indem die Individuen auf diese Ansprüche antworten und sie für ihre eigenen halten, folgen sie der (Selbst-)Regierungsweise eines neoliberalen Subjekts. Dieses Subjekt fokussiert auf seine individuelle Leistungssteigerung, lernt zwischen den besten Optionen auszuwählen, kalkuliert die Balance seiner Kräfte und Reserven und seiner Wünsche und Möglichkeiten, lernt das Privat- und Familienleben mit den Arbeitsanforderungen zu verbinden und in die verschiedenen Lebensphasen planbar $\mathrm{zu}$ investieren. Zu diesen Selbststeuerungsfunktionen gehört auch, das Humankapital möglichst effektiv, langfristig und mit hohen Renditen einzusetzen. In dieser Hinsicht steht das neoliberale Subjekt vor der Neu-Definition der eigenen Gesundheit als »erlernbarer subjektiver Kompetenz.« (Mönch 2018: 285)

Gesundheit kann als Eigenkapital gebraucht werden, um die oben benannten Ziele zu erreichen. Mehr noch, sie kann selbst optimiert und gestärkt werden, damit sie zur besseren Wettbewerbsfähigkeit verhelfen kann (vgl. Brunnett 2018:338). Dadurch allerdings, dass das neoliberale Subjekt selbst für seinen Erfolg haften muss, ist es im gleichen Maße auch für sein Misslingen verantwortlich: »Überforderung oder Scheitern werden nun individualisiert, Versagen des Einzelnen wird als persönliches Scheitern am Diktat der Selbstoptimierung gelesen.« (Flick 2013: 
32) Die Individualisierung der Erkrankung zeitigt zugleich auch neue Normalisierungsprozesse. Indem nicht-kranken, d.h. normalen Arbeitnehmern im öffentlichen Diskurs unterstellt wird, dass sie mit den an sie gestellten Arbeitsanforderungen klarkommen, werden alle anderen zu Sonderfällen und müssen dementsprechend behandelt werden (vgl. Voswinkel 2017: 89). Das gleiche gilt auch für die psychische Gesundheit: "Psychisches Leid ist individuelles Leid und soll auch ebenso behoben werden.« (Flick 2013: 32)

Vor dem Hintergrund der Individualisierung von Gesundheit und der (Neu-)Normalisierung der Arbeitsprozesse werden die Burnout-Betroffenen als diejenigen neoliberalen Subjekte wahrgenommen, die ihren Ansprüchen an Effizienzsteigerung und Ökonomisierung, in öffentlicher oder privater Sphäre, nicht gerecht geworden sind. Diese Vorstellung zieht zumindest zwei direkte Konsequenzen nach sich. Die Burnout-Betroffenen müssen sich zum einen permanent für ihr Versagen rechtfertigen. Zum anderen erfahren sie neue Barrieren und Vorurteile bei dem Versuch der Wiedereingliederung in das Arbeitsleben. Werden sie im ersten Fall mit der Unzufriedenheit über die eigenen Leistungen konfrontiert, die für sie nicht die entsprechende Belohnung gebracht haben (vgl. Engelbach \& Haubl 2017: 119f), so erleben sie im zweiten Fall auf jeweils unterschiedliche Weise Stigmatisierungen: "Leiden Arbeitnehmer an psychischen Überlastungen, dann kommen zu den dadurch verursachten Leistungsdefiziten noch die Angriffe auf ihren Selbstwert hinzu, die von vorurteilsbeladenen Kollegen und Vorgesetzten ausgehen.« (Haubl 2017: 155) Das entscheidende Merkmal bleibt in beiden Fällen unverändert: Den Subjekten wird mitgeteilt, dass ihnen nur durch noch höhere Leistungen, in Form von Rechtfertigung eigener Leistungen oder Abgrenzung gegen fremde Angriffe, ihr gesellschaftlicher Platz garantiert werden kann.

Die oben genannten Leistungsansprüche basieren im Neoliberalismus grundsätzlich auf einem Tauschverhältnis »zwischen einer $\mathrm{zu}$ erbringenden Leistung der Arbeitskraft und einer Gegenleistung der Arbeitsorganisation, nicht nur, aber hauptsächlich ausgedrückt in Geld bzw. materiellen Gratifikationen.« (Kratzer et al. 2015: 49) Durch das Verkaufen bzw. Umtauschen des Humankapitals, mithin auch der psychischen Fähigkeiten, verhandelt das neoliberale Subjekt auf dem Arbeitsmarkt. Es wird allerdings nicht nur zum Austausch seiner Fähigkeiten und Kompetenzen gefordert, sondern auch zu deren Erweiterung und der Bemeisterung seines Selbst, zur sog. Selbstverwirklichung. Dieser neue Anspruch an das neoliberale Subjekt »artikuliert sich erwerbsarbeitsbezogen vor allem in Erwartungen von Beschäftigten im Hinblick auf eine ssubjektivierende` Arbeitsgestaltung, oft im Sinne von Interessantheit, Abwechslungsreichtum, Kreativität und Gestaltungsfreiräumen (in) der Arbeit.« (a.a.O.: 77) Der daraus resultierende Druck zur Selbstverwirklichung und Arbeits-Subjektivierung führt zur Entwicklung der bereits beschriebenen interessierten Selbstgefährdung, bei der sich »Beschäftigte gesundheitlich >gefährden`, um beruflich leistungsfähig zu sein.« (Flick 2013: 15 [Herv. 
i. O.]) Damit wird das neoliberale Subjekt auf eine doppelte Weise zum Selbstregieren geführt: Indem es den äußeren Leistungsansprüchen und gleichzeitig den verinnerlichten Selbstverwirklichungsansprüchen folgt.

\subsubsection{Effekte doppelter Selbstregierung - Anknüpfung an Präventionsdispositiv}

Um den Facetten der oben umschriebenen doppelten Selbstregierung konkreter nachzugehen und einige Konturen des Präventionsdispositivs im Hinblick auf die sich wandelnden Arbeits- und Selbstregierungsformen klarer zu umreißen, werden im Folgenden die Ergebnisse zweier Metaanalysen zusammengeführt und diskutiert.

Die erste Metaanalyse geht auf die Untersuchung der Selbstverhältnisse von Beschäftigten ein und beleuchtet an ausgewählten Beispielen, wie die Praxis der Selbstverwirklichung (oder Selbstsorge) »im Umgang mit den sich wandelnden Anforderungen aussieht.« (Flick 2013: 21)

- So zeigen die Ergebnisse zum einen, dass die Beschäftigten zwei Formen der Anerkennung als für sich relevant deuten: »Der danach als fürsorglich und bedürftig anerkannt zu werden, und der danach als leistungsstarkes und autonomes (Arbeits-)Subjekt Anerkennung zu finden.« (a.a.O.: 197) Dabei wird in der beruflichen Sphäre die Leistungsstärke, in der privaten Sphäre dagegen die Bedürftigkeit mehr zum Ausdruck gebracht (vgl. a.a.O.: 198). Um sich beruflich $\mathrm{zu}$ realisieren, brauchen die Subjekte eine Anerkennung, die auf ihrer Leistung beruht. Um sich privat zu realisieren, sollte die Bereitschaft zur Fürsorge und die Akzeptanz eigener Verletzlichkeit durch die anderen anerkannt werden. Die Arbeitssphäre wird so von den Subjekten als Ort gedeutet, an dem sie Kämpfe austragen können, wobei die private Sphäre als Rückzugs- und Heilungsort fungieren soll, wo die Wunden der Arbeitswelt heilen sollen. Darin wird die primäre Orientierung an der Arbeit sichtbar, die das private Leben $\mathrm{zu}$ bestimmen sucht, und nicht umgekehrt.

- Als zweites Ergebnis hat die Studie nahegelegt, dass die Befragten mit dem Gefühl der Ohnmacht konfrontiert sind, indem sie sich Anforderungen ausgesetzt fühlen, denen sie nicht gewachsen sind (vgl. a.a.O.: 203). Dieses Gefühl resultiert aus der Unmöglichkeit, die Versprechen des selbstunternehmerischen Handelns einzulösen (vgl. ebd.). Die immer komplexer werdenden Aufgaben können die Beschäftigten nie vollständig bearbeiten und bewältigen, was mit dem Versprechen der Selbstverwirklichung, das den neoliberalen Subjekten vermittelt wurde, kollidiert. So wachsen auf der einen Seite die Möglichkeiten zur Selbstverwirklichung, auf der anderen Seite entzieht sich diese den Subjekten mit jeder neuen Leistung, die nie ihr Ziel erreicht, sich zu vervoll- 
ständigen, sich völlig zu realisieren. So kann, paradoxerweise, das permanente Ohnmachtsgefühl die Idee befördern, dass »wenn man nur an sich selbst arbeitete, würde darin ein letzter Handlungsspielraum liegen.«(a.a.O.: 204)

- Schließlich, anknüpfend an die zwei vorherigen Ergebnisse, hat die Studie das Entstehen eines funktionalen und produktiven Selbstverhältnisses nahegelegt, das im Weiteren als Selbstimmunisierung gedeutet werden kann (vgl. a.a.O.: 255). Indem die Beschäftigten große Anforderungen nicht bewältigen können und mit dem Gefühl von Frustration und Ohnmacht konfrontiert sind, suchen sie sich »Aktionsfelder, in denen sie garantierte Selbstwirksamkeit erleben.« (Ebd.) Indem das Bedürfnis nach Anerkennung eigener Leistungen nicht in den vorgegebenen Arbeitsfeldern vollständig eingelöst werden kann, wird dieses in Bereichen gesucht, wo die passenden Möglichkeiten dazu bestehen, wie z.B. Sportleistungen oder Arbeitsleistungen, die nicht unbedingt geleistet werden müssen (vgl. a.a.O.: 256). Erst durch die Bewältigung selbst justierter Leistungsniveaus können sich die Beschäftigten mit Wertschätzung begegnen und anerkennen. In der neoliberalen Regierung des Selbst zählt die Leistung, egal, in welcher Form und an welchem Ort sie auftritt.

Die zweite Metastudie führt eine weitere Analyseebene in die Diskussion der Selbstregierungsverhältnisse neoliberaler Subjekte ein. Hier hat sich die Studie darauf konzentriert, wie die Behandlung von arbeitsbedingten psychischen Störungen durch die Therapeuten vollzogen wird und insbesondere darauf, wie die letztgenannten diese Störungen identifizieren und therapeutisch interpretieren. Drei Deutungsmuster konnten hier beobachtet werden.

- Einerseits beinhaltet die meist vorgekommene (Um-)Deutung der Psychotherapeuten »eine Infragestellung der Arbeitserzählung, was mit einer gleichzeitigen Irrelevanzsetzung arbeitsbezogener Themen einhergeht.« (Flick 2017: 224) Indem bei diesem Deutungsmuster die geäußerten Arbeitsbelastungen »bereits als Ausdruck der Pathologie betrachtet [werden]« (ebd.), werden die äußeren Umstände und gesetzten Arbeitsbedingungen relativiert und als irrelevant interpretiert. Worauf es hingegen ankommt ist ein besseres professionelles Wissen und ein gesunderes Verhältnis zur eigenen Arbeit (vgl. a.a.O.: 225), alles Kompetenzen, an denen die Beschäftigten selbst arbeiten müssen.

- Wenn die Arbeitsverhältnisse doch noch als eine Belastungsquelle zugelassen werden, folgern die Psychotheraputen nicht selten daraus, »dass es nur Verstrickungen sein können, die jemanden freiwillig in solch belastenden Arbeitsbedingungen verweilen lassen.« (a.a.O.: 226) Es wird daher erwartet, dass jeder, der sich unwohl und belastet an seinem Arbeitsplatz fühlt, diesen wechseln oder ihn umzugestalten versuchen würde. Wer ihn hingegen behält, der sucht laut den Therapeuten »aus neurotischen Gründen etwas für 
sich in diesen belastenden Bedingungen: >Wer bleibt, ist krank« (a.a.O.: 227 [Herv. i. O.]), was zur Normalisierung arbeitsbedingter Erkrankungen, wie das Burnout-Syndrom, führen kann.

- Bei dem letzten (Um-)Deutungsmuster werden die prekären Umstände von den Psychotherapeuten anerkannt. Allerdings sollen sich die Beschäftigten bei ihrer Therapie mit sich selbst befassen und die Tatsachen, die sie belasten, einfach akzeptieren lernen. Statt sich dagegen zu wehren, sollen sie bei sich selbst anfangen und die eigenen Potentiale suchen, um »sich zukünftig besser abgrenzen zu können.« (a.a.O.: 229) So transformiert dieses Deutungsmuster äußere Belastungen »in interne Möglichkeitsräume« (ebd.) und verschiebt das Interesse von dem Sozialen auf die Subjekte. So wird durch die Therapie die ohnehin präsente Selbstbezüglichkeit »nun auch für diejenige Patientengruppen, die in ihren subjektiven Krankheitstheorien ihre Arbeitsbedingungen in den Blick nehmen, verstärkt.« (a.a.O.: 231)

Die zwei Metaanalysen haben die Macht-Effekte der doppelten Selbstregierung von beiden Seiten beleuchtet, und zwar am Beispiel der inneren Selbstverwirklichungsanforderung (als Selbstdeutung von Anforderungen bei der ersten Metaanalyse) und am Beispiel einer Leistungsanforderung, die auf die Arbeitssubjekte von außen hinzukommt (als (Um-)Deutungen arbeitsbezogener Erkrankungen in der zweiten Metaanalyse). So gesehen entsteht in den neoliberalen Arbeitsverhältnissen bei den Subjekten ein doppelter Effekt: zum einen kommt es zu einer »Dethematisierung überindividueller Strukturen« (Flick 2013: 298), zum anderen wird ihnen eine »Illusion von Autonomie und Handlungsfähigkeit« vermittelt (Flick 2017: 231). Gegen diese Befunde richten sich andere Studien, die an den Selbst- und Fremdansprüchen von Beschäftigten zweifeln lassen. Nick Kratzer et al. sind in ihrer Studie zu den Legitimationsproblemen der Beschäftigten zum Schluss gekommen, dass die neoliberalen Werte »kaum über eine legitimatorische Basis unter den Beschäftigten [verfügen], ihre Basiswerte werden nicht zu Handlungsorientierungen der Beschäftigten.« (Kratzer et al. 2015: 395) Die Subjekte scheinen daher aus dieser Perspektive nicht den neoliberalen Ansprüchen ausgesetzt zu sein und folgen diesen auch nicht. Gleichzeitig, so Kratzer et al., werden sie bei den Beschäftigten auch nicht thematisiert: »Bestimmte Ansprüche und Erwartungen, die enttäuscht werden könnten, werden gar nicht (mehr) an die betriebliche und gesellschaftlichökonomische Ordnung gestellt." (Ebd.) Ob sich daraus folgern ließe, dass die Beschäftigten als neoliberale Subjekte die Anforderungen bereits soweit verinnerlicht und verselbstständigt haben, dass sie gar nicht mehr das Bedürfnis haben, diese als programmatische Leitsätze bewusst zu artikulieren, bleibt abzuwarten. Der leere Ort der Legitimationsbasis entzieht sich sowohl einer direkten Kritik (vgl. ebd.) als auch einem direkten Widerstand. Was nicht zu identifizieren und zu benennen ist, 
kann auch nicht kritisiert oder umgeworfen werden. Gerade an diesem Ort kann das Präventionsdispositiv am besten ansetzen.

Die Prävention gesundheitsgefährdender Arbeitsverhältnisse und Verhaltensweisen erscheint in erster Weise als eine Notwendigkeit. Sie ist an die, berufsbezogene oder private, Möglichkeitsräume neoliberaler Subjekte gebunden und erscheint allen Beteiligten als selbstverständlich: »Eine Verweigerungshaltung in Bezug auf Prävention wirkt nicht nur wie eine sEntscheidung gegen den Zeitgeist<, sondern wird als >individuelles Unvermögen gedeutet, mit den Anforderungen Schritt zu halten` (Jürgens 2009: 221).« (Mönch 2018: 298) Daher kann das Präventionsdispositiv am einfachsten dort operieren, wo es nicht in Frage gestellt wird und wo es sich unter dem Mantel einer von den Subjekten selbst erwünschten präventiven Hilfe verbergen kann. Indem sich nämlich die Subjekte nicht auf strukturelle, sondern individuelle Probleme beziehen, indem sie unter doppelten Leistungsansprüchen leiden und diese auf sich selbst beziehen, und indem sie neoliberale Denk- und Handlungsweisen nicht hinterfragen bzw. um sie nicht wissen, stellen sie das ideelle Raster des Homo oeconomicus dar, das die »Kontaktfläche zwischen Individuum und Macht« (a.a.O.: 257), und somit den Bereich der Regierungstechnologien, abbildet. So lassen sich die verschiedensten Verhaltensweisen, die in den Arbeitsbeziehungen auftreten, als Versuche deuten, neue, auf präventiver Rationalität aufbauende Regierungsverhältnisse zu etablieren.

\subsection{Problematisierung der Gesundheit}

Die wachsende Sorge um die psychische Gesundheit hat ihre historischen Entwicklungsphasen. Das folgende Kapitel soll daher die Art und Weise diskutieren, wie Gesundheit zum Gegenstand öffentlicher Problematisierung geworden ist und welche historischen Wendepunkte dabei zu beachten sind. Des Weiteren wird sich die Aufmerksamkeit auf die Psyche als einen neuen Ort der Machtkämpfe richten, der im Zuge einer allgemeinen Psychopolitik neue Bedeutung gewinnt. Schließlich wird das Burnout-Syndrom als eine Regierungstechnik des Präventionsdispositivs diskutiert, was die Frage eröffnet, inwiefern die psychischen Zustände von Subjekten auch in Gegen-Kämpfen angesetzt werden können.

\subsubsection{Gesellschaftliche Problematisierung psychischer Gesundheit}

Die Thematisierung der psychischen Gesundheit als eines gesellschaftlichen Problemfeldes lässt sich nicht eindeutig geschichtlich verorten und umreißen. In der Geschichte westlicher Gesellschaften hat die psychische Gesundheit unterschiedliche Ausprägungen und Facetten angenommen, je nachdem, in welchem Zusammenhang sie zu den jeweils prägenden sozialen und kulturellen Wandlungen stand. Als eine mögliche Option, ihre geschichtliche Problematisierung 
kurz aufzuzeigen, bietet sich die Beschreibung paradigmatischer Veränderungen an, die das Problematisieren von seelischen, und später psychischen, Zuständen erfahren hat. Im Folgenden werden fünf solche Veränderungen beschrieben:

- Eine erste Kontur der Vorgeschichte des psychischen Diskurses lässt sich in der antiken Sorge um Melancholie erkennen. Für den antiken Menschen stellte eine depressive Stimmung keinen Grund für Beunruhigung dar, denn sie gehörte zu seinem Wesen. Die schwarze Galle (griech. melaina chole) wurde als ein eigenständiger Körpersaft angesehen, welcher, im richtigen Mischungsverhältnis mit den anderen Säften, »konstitutiv für gesunde Menschen « war (Abels 2015: 93). Dementsprechend wurde die Melancholie von den antiken Philosophen nicht als eine Krankheit bezeichnet (vgl. ebd.), sondern als ein Leiden der Seele, das »aus ihrer falschen Führung resultiert. (Abels 2015: 117) Auch deswegen haben sich die Interventionen vornehmlich auf die Beruhigung der Seele durch Meditationen, Exerzitien und Gelassenheitsübungen konzentriert.

- Im Mittelalter lässt sich die Sorge um die Seele im Begriff der Acedia (griech. akedeia) zusammenfassen, mit der im übertragenen Sinne die »Müdigkeit des Herzens« (a.a.O.: 119) bei den Mönchen bezeichnet wurde. Es handelte sich hierbei vornehmlich um eine religiöse Versuchung der frühchristlichen Mönche, sie von dem Gebet und von dem religiösen Leben in den Klöstern abzuhalten, sie in Schwermut, Müdigkeit und Trägheit fallen zu lassen und somit die christliche Glaubenspraxis zu vernachlässigen (vgl. ebd.). Die an die Sorge um die müde Seele anknüpfenden Praktiken haben sich vor allem als Aufforderung zur physischen Arbeit (vgl. ebd.), als Gedankenkontrolle durch die »Einübung des Todes« (a.a.O.: 121) oder als Buß- und Beichtpraktiken, die vor allem mit Wiedergutmachung verbunden waren, manifestiert (vgl. a.a.O.: 128f).

- Mit dem Erscheinen der Aufklärung fand eine Transformation der Traurigkeit, der Melancholie, statt. Diese Transformation konnte dadurch geschehen, »dass sich die entstehende Psychiatrie sowie die populären Versuche, die Melancholie mithilfe einer $>$ Erfahrungsseelenkunde zu erfassen, zunehmend auf das Konzept der >Psyche< stützen, das in der Folge den Rekurs auf die Seele verdrängen wird.« (a.a.O.: 148 [Herv. i. O.]) Durch eine solche Verschiebung konnte das Innere des Menschen »stärker zum Objekt der Intervention werden, zum Ziel und zum Maßstab der Bemühungen und Techniken zur Selbstveränderung ( (ebd.). Im Vergleich zur Feudalgesellschaft, wo die Seele noch »den Bezugspunkt für die Auseinandersetzung mit dem Selbs [bildet], steigt nun die Psyche zum hegemonialen Deutungsschema von Innerlichkeit auf.« (a.a.O.: 149) Diese psychologischen Deutungsmuster entstehen also erst in dem Moment, »in dem mit der Aufklärung auch die Idee des modernen Individuums entsteht, das alle theologischen Bestimmungen abgelegt hat.« (Sonntag 1988 zit.n. Abels 2015: 148) 
- In der Moderne findet ein weiterer Paradigmenwechsel mit den Freud'schen Untersuchungen des menschlichen Bewusstseins und Unterbewusstseins statt. Die an ihn anlehnende Entwicklung und Verbreitung von Praktiken der Psychoanalyse und Psychotherapie haben den »im psychologischen Sinne sgläsernen Menschen« « produziert (Meschnig 1993: 13 [Herv. i. O.]) und zu einer »massiven Diskursverschiebung in der Konzeption des Selbst« geführt (Abels 2015: 179).

- Im 20. Jahrhundert ließen sich die Perioden der Problematisierung psychischer Gesundheit etwas klarer abgrenzen. So zeigt Patrick Kury, dass das erste Konzept, welches den Stress und mithin die psychische Gesundheit zum ersten Mal als ein "gesamtgesellschaftliches Anliegen« (Kury 2012: 297) thematisierte, die sog. Neurasthenie war. Anders als bei der dazu komplementären Hysterie, handelte es sich um eine psychische Störung bzw. Belastung. ${ }^{63}$ Bei der Behandlung dieser Störung ging man davon aus, dass »im Körper dynamische Vorgänge wirken, die ein innerorganisches Gleichgewicht anstreben. Zentral dabei ist der Terminus der >Homöostase`, der die Fähigkeit des Organismus bezeichnet, selbstregulierend zu wirken. "(Seyle 1957: 24f zit.n. Kury 2012: 28 [Herv. i. O.]) Der menschliche Organismus, so dachte man, funktioniert wie ein Stromkreislauf und nach dieser Erkenntnis wurde er auch behandelt (vgl. a.a.O.: 296). Im Gegensatz zur Neurasthenie ging das zweite Konzept der sog. Managerkrankheit »von einer stärker mechanistischen Vorstellung des Körpers aus. Diese eng mit der Entwicklung der Industrialisierung verbundene Konzeption deutete den menschlichen Körper als eine Art Maschine.«(a.a.O.: 29) Besonders im Deutschland der 50-er Jahren stellte diese psychische Krankheit »einen direkten Zusammenhang her zwischen Herzkreislauf, Automatisierung und den gestiegenen Belastungen durch Wiederaufbau und Wirtschaftswachstum nach dem Zweiten Weltkrieg.«(a.a.O.: 296) Dem dritten Konzept des Burnouts gingen die biochemische (vgl. Abels 2015: 195ff) und epidemiologische (vgl. a.a.O.: 206) Phase des Depressionsdiskurses voraus, die das Bild von der Depression geprägt und das Phänomen psychischer Störungen wie Burnout-Syndrom medikalisiert und individualisiert haben. Dadurch wird das Burnout-Syndrom »in Analogie zum Allgemeinen Anpassungssyndrom und Stress als Störung der

63 Die Entwicklung unterschiedlicher Konzepte von Geisteskrankheiten wurde auch von der Diskussion um geschlechtliche Differenzen begleitet. Hysterie, z.B. assoziierten die Mediziner mit weiblichen Reaktionen, wobei die Neurasthenie als eine männliche Stressreaktion verstanden wurde. Wie die Diskussion um Neurasthenie zeigt, legten viele Autoren diese Diagnose »als männliches Komplement zur Hysterie aus und in der Tat kann man dies an den prozentualen Häufigkeiten der jeweiligen Diagnosen bei Männern und Frauen verfolgen. Galt die Sorge der Mediziner bis dato vor allem den hysterischen Frauen, so kamen mit der Neurasthenie nun auch vermehrt die Männer in den Fokus medizinischer Überlegungen.« (vgl. Shorter 1994: 377 zit.n. Abels 2015: 173) Damit wird ein Forschungsdesiderat umrissen, das an wissenschaftlichem Interesse gewinnt (vgl. Pfütsch 2017). 
Botenstoffe, also des biochemischen Informationsaustauschs, begriffen.« (Kury 2012: 296)

Die oben aufgeführte Periodisierung soll nicht eine zeitliche oder geschichtliche Abfolge abbilden, sondern vielmehr aufzeigen, wie sich die Problematisierung psychischer Gesundheit gewandelt und welche unterschiedlichen Formen sie angenommen hat. Die vielschichtigen Facetten dieser Entwicklung bestimmen nämlich auch die Entstehung und Funktionsweise des Präventionsdispositivs. So bezieht sich dieser z.B. auf die Freud'schen Untersuchungen, dank welchen die moderne Psychologie das Postulat verbreiten kann, »dass das Subjekt von verborgenen Kräften bestimmt wird, die ihm nicht bewusst sind. (Meschnig 1993: 12) Um diese zu benennen und interpretieren zu können, bedarf es eines Expertenwissens. Ein Experte kann jedoch nur dann etwas bewerkstelligen, wenn der Mensch eine Aussage über sich macht, d.h. etwas gesteht - worin die mittelalterliche Sorge um die Seele zum Ausdruck kommt. Indem die Buß- und Beichtpraktiken den Weg zu den Technologien des Geständnisses gebahnt haben, wurde »das Vordringen in die geheimsten Nischen des Individuums, die Kenntnis seiner verborgensten Wünsche und die Transformation eines kulturellen Zwanges in ein individuelles Gefühl der Freiheit« möglich (ebd.). ${ }^{64}$ Auch die spätmodernen industriellen und kapitalistischen Entwicklungen, die die Thematisierung stressbedingter Störungen begleitet haben, zeigen, wie sich die Psyche unaufhaltsam zur Projektionsfläche gesell-

64 Den Anfang dieser Technologien und deren Wandlungen verortet Meschnig in die vormodernen ökonomisch-politischen Veränderungen. So wurde bspw. durch die zunehmende Individualisierung und Auflösung traditioneller Bezüge das Subjekt aufgefordert, in sich selbst eine Identität zu erzeugen. »Das moderne Subjekt ist nicht stumm. Im Gegenteil: es muss sprechen. Bestimmte Formen des Wissens, wie sie etwa in der Psychologie, der Pädagogik oder auch der Medizin angestrebt werden, verlangen nach einem sprechenden Subjekt. Da aber das Subjekt gleichzeitig nicht mehr weiß, was ihm entgeht, braucht es technische Verfahren und kompetente Fachleute um seine Wahrheit an den Tag zu fördern.« (Meschnig 1993: 84) Meschnig zeigt in seiner Studie auch weitere Modifikationen, durch die die Produktion der Wahrheit seitens des Subjekts die westliche psychische Landschaft geprägt hat (vgl. a.a.O.: 84ff). In ähnlicher Weise untersuchte auch Ingar Abels, wie durch den Depressionsdiskurs »jene Form der Normalisierung genau wirkt, die auffällige Subjekte an einem Set von positiven Wissensbeständen misst, pathologisiert und dadurch erst normalisiert.«(Abels 2015: 80) Die Problematisierung der Depression entlarvt sie dann durch die Hinterfragung der Praktiken, die die depressiven Subjekte hervorbringen, definieren und subjektivieren (d.h. sie zur Selbstführung anleiten): »Mit welchen Techniken soll das Subjekt auf sich einwirken oder auf sich einwirken lassen, um sich als depressives Subjekt zu identifizieren und dann zu verändern? Was sind die genauen Ziele dieser Interventionen, die als Antwort auf eine spezifische ,Sorge um Depressionen gegeben werden? Gibt es in diesem Sprechen ein leises, implizites Raunen darüber, wie Subjekte zu Beginn des 21. Jahrhunderts mit ihren Traurigkeiten und Melancholien umgehen sollen?« (a.a.O.: 81 [Herv. i. O.]) 
schaftlichen Unbehagens und zum Terrain politischer Machtkämpfe entwickelt hat (vgl. Rau 2010: 417).

\subsubsection{Psyche als Ort der Machtkämpfe}

Wie im letzten Teil gezeigt wurde, war die menschliche Seele bzw. Psyche als Gegenstand zahlreicher Thematisierungen in der Geschichte westlicher Gesellschaften durchaus präsent. Die Individuen haben dadurch gelernt, über ihr psychisches Wohlbefinden nachzudenken, es als öffentliches Thema zu präsentieren und die erlebten Befindlichkeiten vor sich und vor den anderen $\mathrm{zu}$ gestehen. Mit dieser Verselbstständigung des psychischen Diskurses wurden die Individuen zu immer größerer und umfangreicherer Transparenz und Offenheit geführt. Die psychische Gesundheit, gleichwie ihre Negation, die Krankheit, lassen sich dadurch »als soziale und zugleich diskursive Konfliktfelder « betrachten (Poczka 2017: 17). Sie verbinden in sich ebenso individuelle Eigenschaften wie sozial und diskursiv eingeprägte Verhaltensweisen, Normen und Erwartungen. Das gegenwärtige Verständnis von Psyche kann daher als »ein materialisiertes Ensemble von modernen Konzepten und Praxen des Selbst verstanden werden, das es dem Einzelnen erlaubt, sich praktisch und sinnhaft als individualisiertes Subjekt zu sich und anderen ins Verhältnis zu setzen und dieses Verhältnis durch eine reflektierte Arbeit am Selbst führen und verändern zu können.«(Rau 2010: 179) Die Arbeit an sich selbst korrespondiert in diesem Sinne mit den neoliberalen Ansprüchen an die Subjekte, sich selbst optimal zu organisieren und den inneren wie äußeren Anforderungen entsprechend $\mathrm{zu}$ handeln. In ihrer Studie zur Psychopolitik geht Alexandra Rau aus einer der westlichen Gesellschaften gestellten Diagnose der »Subjektivierung von Arbeit« (vgl. Moldasch \& Voß 2002a zit.n. Rau 2010: 9) heraus. Die Ursachen ihrer Entstehung sieht sie einerseits darin, dass Beschäftigte zur Selbststeuerung von Arbeitsprozessen aktiviert werden und »einem Erfordernis zur Selbst-Organisation und SelbstÜberwachung ausgesetzt sind« (vgl. Lohr 2003: 511 zit.n. Rau 2010: 10), und andererseits darin, dass sie, als Subjekte der Arbeit, "sowohl sinnvolle Arbeitsinhalte als auch die Gestaltung der eigenen Arbeitsbedingungen « einfordern (Rau 2010: 11). Diese, mit dem fortschreitenden kapitalistischen Strukturwandel einhergehenden Anforderungen an die Selbstsorge, werden von manchen Forschern als die Wirkung eines Aktivierungsdispositivs umschrieben (vgl. Gilles 2008; Abels 2015). Im Zentrum dieses Dispositivs stehen disparate Aktivierungsstrategien, mit denen komplexe Technologien der Selbst-Aktivierung beschrieben werden, »die darauf zielen, die eigenen physischen, psychischen und sozialen Fähigkeiten als aktivierbare Ressource zu erkennen." (Abels 2015: 255) Beispiele solcher Strategien lassen sich »innerhalb der Re-Organisierung der öffentlichen Verwaltung, in ManagementRatgebern und den Appellen an eine aktive Gestaltung der Lebensführung und der Gesundheit finden.« (a.a.O.: 244) Mit ihrer Hilfe wird die Psyche nicht nur 
zum »Objekt von Interventionen, die sie lediglich leistungs- und widerstandsfähiger und das Individuum damit arbeitsfähiger machen« (a.a.O.: 276f), sondern zu einer offenen Arena, »in der Individuen Themen der Selbstbestimmung und des Glücks verhandeln.« (Abels 2015: 277; vgl. auch Duttweiler 2004) So gedeutet wird die Psyche zu einem Kampf-Terrain, »auf dem und durch das regiert werden kann, wo Verletzungen (Scham, Entwürdigung, Verlust von Integrität, Desidentifikation usw.) wie auch Ermächtigungen (Anerkennung, Befreiung usw.) statt haben; wo all dies ungleich verteilt und normativen Vorstellungen unterworfen wird; wo es ein Begehren nach dem Selbst gibt, das ausbeutbar ist (vgl. Butler 2001: 12). (Rau 2007: 43) Und es ist dieses Terrain, auf dem sich die Psyche mit den Regierungstechnologien verschränkt und zu einer allgemeinen Psychopolitik wird.

Psychopolitik ließe sich als ein Set von alltäglichen Strategien und Praktiken deuten, »die sich auf die Führung und Bearbeitung der Technologien des Selbst beziehen und im Modus der Psyche mit Herrschaftstechniken vermitteln." (Rau 2010: 295) Mit diesem Begriff wird eine Regierungsweise bezeichnet, die heutzutage den Aufstieg psychologischer Rationalitäten markiert, welche Befreiung, Normalität und Orientierung verheißen und zur »Vervielfältigung des Lebens, der Möglichkeiten des Fühlens und Wahrnehmens oder schlicht [zur] Möglichkeit, anders zu leben« führen sollten (Abels 2015: 192). Gleichzeitig ist durch die Verschränkung von Regierungstechnologien und popularisierten psychischen Geständnissen die menschliche Psyche deutlich durchsichtiger und lesbarer, und damit auch verletzbarer, geworden. Die Psyche funktioniert daher nicht nur als eine »reflektierte Praxis von Menschen« (Foucault [1978] 2004: 359 zit.n. Rau 2010: 180), sondern auch als ein Terrain, von dem ausgehend und um es gruppiert »Kämpfe geführt werden (können).«(a.a.O.: 417) Die Individuen können nämlich ihre innersten Bewegungen und Sehnsüchte nur dann gestehen, wenn sie in einem diskursiven Feld erst einmal intelligibel erscheinen können. Erst das Benennen und In-Wort-Fassen eines Gefühls oder eines Gedankens macht die psychischen Zustände plausibel und zugänglich. So kann die Psyche als ein diskursiv hervorgebrachtes Problemfeld charakterisiert werden. Und es ist ihr Eintreten »als Diskurs und Praxis auf signifikante, und zwar hegemoniale Weise ins Feld der Macht« (ebd.), die es erlaubt, von Psycho-Politik zu reden. Aus macht- und herrschaftskritischer Perspektive eröffnet sich dann Frage, wie sie als Ort der Subjektformation und gleichzeitig als Ort, wo »Kämpfe um das Selbst « (ebd.) ausgetragen werden, funktioniert. ${ }^{65}$ Die Sub-

65 Rau verbindet das Terrain der Kämpfe, das sie in der Psyche entziffert, mit den emanzipatorischen Anforderungen der feministischen Bewegung. Am Beispiel der Selbsterfahrungsgruppen, in denen die Frauen ihre Erfahrungen der Unterdrückung teilen, demonstriert sie die Programmatik dieser Kämpfe: »Indem Frauen sich in einem gruppendynamischen Prozess zu Psychoexpertinnen ihrer selbst ermächtigen, werden sie im selben Atemzug nicht nur Gesellschaftsanalytikerinnen, darüber hinaus erhält die persönliche, therapeutische Selbstexploration den Status einer politischen Strategie, eines politischen Kampfmittels, das ver- 
jektformation ebenso wie die Kämpfe um das Selbst knüpfen unmittelbar an die neoliberale Regierungsrationalitäten an, die sich auf zwei Ebenen etabliert haben:

- Auf der strukturellen und institutionellen Ebene bildet sich im 18. und 19. Jahrhundert eine liberale Rationalität der Gesundheitsregierung, deren Entwicklung "auf einer andauernden Rationalisierung der Verfahren [basierte], durch die die Menschen $\mathrm{zu}$ einem bestimmten gesundheitsbezogenen Verhalten angeleitet und Regierungen $\mathrm{zu}$ bestimmten gesundheitspolitischen Strategien motiviert und für diese legitimiert wurden.« (Poczka 2017: 421)

- Auf der individuellen Ebene kommt es im 20. Jahrhundert und am Anfang des 21. Jahrhunderts zur schrittweisen Psychiatrisierung des Alltags (vgl. Castel et al. [1979] 1982). Das Funktionieren dieser Rationalität offenbart sich in dem ständigen Praktizieren psychischer Hygiene durch Gespräche über eigene Gefühle, Gedanken, Träume, Vorstellungen, Sehnsüchte usw.

Beide Rationalitäten lassen sich als Bestandteile neoliberaler Regierungsregime bezeichnen, welche einerseits eine institutionelle Veranlagung benötigen und andererseits auf Wissensproduktion - durch individuelle Geständnisse - angewiesen sind. Ein kennzeichnender Aspekt neoliberalen Regierens ist im Weiteren die permanente Differenzierung: »Die liberale Regierung darf, um effektiver zu sein, vor allem nicht unnötig regieren. Dafür ist eine Unmenge an Differenzierungen nach dem Kriterium der optimalen Regierbarkeit des jeweiligen Problems und der für jedes Problem geeignetsten Techniken vorzunehmen.« (Poczka 2017: 434) Daher muss auch das psychische Befinden kontinuierlich differenziert werden, um für jede neue Diagnose eine passende Heiltechnik anwenden zu können und die Individuen auf sie zu verweisen. So gewinnen sie nicht nur das Gefühl, dass sie um ihre Störungen und Erkrankungen wissen und dass diese heilbar sind, sondern dass sie mit ihren Zuständen normal leben und sie für selbstverständlich und natürlich halten können. Die neoliberale Psychopolitik setzt also solche Mechanismen ein, »die es für jedes Individuum zum persönlichen Nachteil machen, ihrer Rationalität zuwider zu handeln." (Ebd.) Eine solche Differenzierung kann beispielhaft an dem Problematisieren des Burnouts veranschaulicht werden.

spricht, die patriarchale `Unterdrückung zu besiegen «. Wenn folglich das Private für politisch erklärt wird, bedeutet dies nicht zuletzt zu erkennen, dass sich das politische in der psychischen Struktur (wie im Psychosexuellen) spiegelt und verdichtet. Es wird damit zum Ort und zum Modus einer feministischen widerständigen Praxis. [...] Politisch zu sein und sich die Welt gestaltend anzueignen heißt also auch, sich selbst und die Beziehung zu anderen authentisch zu leben.« (Rau 2010: 288 [Herv. i. O.]) 


\subsubsection{Burnout als Regierungstechnik des Präventionsdispositivs}

Anlehnend an die neoliberale Taktik der Differenzierung zählt Burnout zu einer Störung, die nicht eindeutig definiert werden kann. Dem liegt die Tatsache zugrunde, dass sich seine Definition in den meisten Fällen »auf einzelne Personen und nicht auf das Verhalten einer ganzen Kohorte im Zeitverlauf« bezieht (Burisch 2014: 239). Damit dürfte »die Zahl inoffiziell-persönlicher Definitionen bzw. Varianten [...] annähernd der Gesamtzahl Betroffener entsprechen.« (Hillert \& Marwitz 2006: 280) Gerade wegen der personalisierten und also individuell differenzierten Beschreibung des psychischen Befindens beim Burnout ließe sich diese Störung als eine neoliberale Regierungsform entschlüsseln. Bei dieser psychischen Erscheinung ist es der Betroffene selbst, der seinen Zustand beschreiben und deuten muss, trotz der Verwirrung, die damit einhergeht: »Er ist Bewohner einer Zwischenwelt. Offiziell ist er nicht krank. Er fühlt sich aber auch nicht gesund. Wie soll er also diesen Zustand interpretieren und wie soll er seine Beschwerden einordnen?" (Brendt \& Sollmann 2012: 23) Wird dann die Störung als individuelle Angelegenheit präsentiert, braucht sie auch auf der individuellen Ebene eine entsprechende Behandlung. An dieser Stelle knüpfen die Praktiken des Präventionsdispositivs an.

Wie die Forscher zeigen, fokussieren die meisten Ansätze zur BurnoutPrävention auf »Verhaltensprävention $<$ - also auf Modifikation der individuellen Risikofaktoren: 86 Prozent der Studien bezüglich Prävention von Stress-Belastung und Burnout beziehen sich auf die individuelle Ebene, wobei meist kognitiv-verhaltenstherapeutische Interventionen empfohlen werden (Walter/Krugmann/Plaumann 2012).« (Gross 2016: 260 [Herv. i. O.]) Diese Strategie des Präventionsdispositivs dient im Licht der neoliberalen Leistungslogik zum einen dem Auftrag zur Selbstoptimierung: »Burnout soll von den Betroffenen möglichst zügig, unter Einsatz optimaler Ressourcen und durch den vollen persönlichen Einsatz bearbeitet und gelöst werden." (Heinemann \& Heinemann 2016: 248) Andererseits inkorporiert das Dispositiv der Prävention auch die (möglichen) Versuche der Betroffenen, ihr Burnout als Anerkennung für die vollbrachten Leistungen $\mathrm{zu}$ akzeptieren und Burnout als persönliche Auszeichnung umzudeuten, und zwar so, »dass man jetzt die `Anerkennung für das Arbeitsleid (wenn schon nicht für die früher geleistete Arbeit) sucht - und im Falle der Veröffentlichung einer Burnout-Symptomatik oft auch findet.« (Gross 2016: 263 [Herv. i. O.]) Als eine Regierungstechnik funktioniert dabei auch die dazu notwendige therapeutische Behandlung.

Die psychotherapeutischen Maßnahmen und Verfahren zeitigen unterschiedliche Effekte bei der Behandlung des Burnout-Syndroms. Aus kritischer Perspektive lassen sich Aspekte kontrollierender und disziplinierender Techniken beobachten, die in der introspektiven, auf sich selbst bezogenen Therapie enthalten sind. Christina Sonnenfeld schrieb dazu:»[D]er Zwang zur positiven, und vor allem selbsttäti- 
gen Auflösung von Leiden soll die Seele ruhig stellen. Abwesenheit jeglicher unkalkulierbarer Irritationen, keine Leiden, keine Schmerzen - das ist das Ideal.«(Sonnenfeld [1989] 2016: 33) Damit soll nicht nur die Problematisierung innerer Konflikte und Schwierigkeiten mit der Selbstbemeisterung als Gegenstand der Kritik erscheinen, sondern auch der breitere, gesamtgesellschaftliche Kontext reflektiert werden:

»Eine Fokussierung ausschließlich auf einen dekontextualisierten Homo oeconomicus kann die Individualisierung und Pathologisierung gesellschaftlicher Probleme nur fördern, statt diese Entwicklung zu problematisieren. Daher bleibt die Tätigkeit der Therapeut/innen eine eminent politische - auch und gerade dann, wenn sich Ärzte/Ärztinnen und Therapeuten/Therapeutinnen als >unpolitischeく Experten/Expertinnen verstehen.«(Gross 2016: $265 f$ [Herv. i. O.])

Indem das Präventionsdispositiv als eine Selbstverständlichkeit, und also unproblematisch erscheint und daher keiner Reparatur oder gar Verwerfung bedarf, braucht es eine sensiblere Zugangsweise, um seine Wirkung und seine unterschwelligen Machteffekten zu entfalten. Wird nämlich Burnout als »eine Wirkung neoliberaler Gouvernementalität« (Hillebrand 2013: 131) identifiziert, so gilt es diese Regierungstechnologie zu hinterfragen und ihre Mechanismen genauer auszuloten. Wie bereits erwähnt, finden um die Prozesse der Subjektformierung und der Führung des Selbst mannigfache Kämpfe statt, die von der Psyche aus strukturiert werden (vgl. Abels 2015: 193) und die einen konstitutiven Moment des Regierens darstellen (vgl. Maasen 2011: 9). Es handelt sich dabei um ganz konkrete Kämpfe, die die Individuen in ihren Selbstregierungspraktiken vollziehen: «Kämpfe um Teilhabe, Schutz, Rückzug, die Möglichkeit, jemand anders zu sein, Kämpfe um Solidarität und um die Möglichkeit, nicht identisch sein zu müssen mit sich selbst.« (Abels 2015: 193) Selbst bei Burnout können diese Kämpfe unterschiedlich aussehen. So kann man bspw. »die Selbstbeschreibung als Burnout-Opfer auch lesen als eine subversive Verwendung des rhetorischen Arsenals der neoliberalen Optimierungsgesellschaft.«(Gross 2016: 263) Oder, wird der Burnout als Scheitern an der aktiven Gestaltung des Selbst und an der Sorge um die eigene Gesundheit gedeutet, so erwächst gerade aus diesem Scheitern »das Potenzial, Gesundheit gesamtgesellschaftlich in Begriffen und Konzepten $\mathrm{zu}$ problematisieren, welche politisch über die neoliberale Programmatik hinausweisen.« (Brunnett 2007: 181)

Die Kämpfe um das Selbst können allerding auch auf einer kollektiven Ebene stattfinden. Einer der ersten Versuche eines solchen öffentlichen und kollektiven Kampfes war z.B. die Demonstration der aktivistischen Gruppe Feel Tank Chicago im Jahr 2003. Gekleidet in Pyjamas und Bademantel wollten die Demonstranten die Depression und das feeling bad ihrer neurochemischen Deutungshoheit entziehen und »im Zusammenhang mit neoliberalen Arbeits- und Lebensverhältnissen, mit Homophobie, Sexismus und postkolonialem Rassismus als public feelings [...] poli- 
tisieren. Das Gefühl in einer Sackgasse zu stecken, nicht zu wissen, was zu tun ist, nicht aufstehen zu können oder blockiert zu sein, ist demnach nicht als Krankheitssymptom zu betrachten, sondern als Ausgangspunkt für politisches Engagement und soziale Veränderung.« (Bernet 2012: 189) Diese kollektiven und individuellen "Kämpfe um Subjektivierung« (Rau 2010: 44) gilt es im Rahmen einer kritischen Untersuchung zunächst »als solche überhaupt sichtbar zu machen und sie als solche anzuerkennen« (ebd.), was auch das vorliegende Projekt beabsichtigt hat.

\subsection{Das Denken in Risiken}

Für einen fruchtbaren Boden, auf dem die Prävention gesellschaftlich thematisiert werden und gedeihen konnte, hat die wachsende Problematisierung des Risikos gesorgt. Die Problematisierung des Risikos lässt sich erstens entlang der historischen Entwicklung der Verpflichtungsbeziehungen zwischen dem Staat bzw. der Gesellschaft und dem Individuum beobachten. Zweitens können ihre Formen vor dem Hintergrund der globalen Entwicklungen beschrieben werden. Schließlich lässt sich drittens die Problematisierung des Risikos als ein Diskurs auffassen, der quer $\mathrm{zu}$ den beiden obengenannten Themen liegt. Die drei unterschiedlichen Dimensionen der Risiko-Problematisierung werden in dieser Reihenfolge kurz umrissen.

\subsubsection{Historische Konturen der Risikoproblematisierung}

Die historische Entwicklung der Risiko-Problematisierung folgt drei wechselnden Linien zwischen der individuellen und der gesellschaftlichen Verantwortung für Risiken.

- Die erste Linie fing mit der Notwendigkeit an, wirtschaftlichem Schaden vorzubeugen. Diese Notwendigkeit hat womöglich mit dem Aufbau institutionalisierter Marktbeziehungen sowie mit den Entdeckungen neuer Transportwege im 16. Jahrhundert an Bedeutung gewonnen. Mit der Versicherung von Waren und Gütern sollte eine »Übernahme von Verlustrisiken gegen vorherige Prämienzahlung « erzielt werden (Conze 2018: 43). Wenn die Unsicherheiten, die mit dem ökonomischen Handel verbunden waren, in Risiko verwandelt wurden, konnte das Geschäft auf seine konkreten Fallstricke berechnet und versichert werden: »Denn versichert werden nicht unkalkulierbare Gefahren oder Unsicherheiten, sondern kalkulierbare Risiken. Diese Transformation von Unsicherheit und Gefahr in Risiko ist der Kern des Versicherungsgeschäfts.«(Ebd.) Diese wirtschaftliche Rationalität wurde von einer rechtlichen Auffassung der bestehenden Verträge begleitet. Demnach haben sich die Fragen der Verantwortung für mögliche Risiken mit der Versicherung der Güter und Waren und der rechtlichen Haftung für die entstehenden Übel verbunden, was zu einer 
Entstehung des Dispositivs der Haftung führte, »wodurch jede*r für sich selbst verantwortlich gemacht werden konnte. (Perthus 2016: 28)

- Die zweite geschichtliche Linie hat mit der industriellen Revolution begonnen. Mit ihr haben sich Fragen der Arbeits-, Unfall- und Altersvorsorge auf neue Weise gestellt und drängten den Staat zur Sorge für die beschäftigten Bürgern und ihre Familien. Die sodann entstehenden Versicherungsgesellschaften fungierten als Gesellschaften, »in denen an die Stelle sozialer Unsicherheit für die große Mehrheit der Bürger eine seitens des Staates garantierte allgemeine soziale Absicherung trat. Der Sozialstaat spielte eine fundamentale Rolle, wo es um das >Begrenzen von Risiken ging. " (Castel 2009: 24 [Herv. i. O.]) Damit wechselte die "Sicherheitsfrage vom Problem der >Verantwortlichkeit<, der >Haftung<, zum Problem der >Solidarität<, der >Gesamtschuldnerschaft<." (Ewald [1986] 1993: 16 [Herv. i. O.]) An die Stelle des Dispositivs der Haftung tritt somit das Dispositiv der Solidarität bzw. das Dispositiv der sozialen Sicherheit. Dieses Dispositiv erwächst aus der Erkenntnis, dass die Gesellschaft »die Übel selbst erzeugt, die auf ihr lasten und ist daher gezwungen, ihre eigenen Formen der Sicherung zu finden. Die Gesellschaft als Ganzes muss für und gegen sich selbst einen Mechanismus, ein Dispositiv einrichten, das beides miteinander vereint und verschränkt: Unsicherheit, Ungewissheit, Leben mit dem Risiko auf der einen und Absicherung, Risikominimierung, Planbarkeit auf der anderen Seite.«(Bohlender 2010: 103f) Mit diesem Dispositiv ist die Prävention gegenüber arbeitsbedingten Risiken »als staatliche Aufgabe behandelt worden, die kollektiv zu lösen ist. Es ist fortan weniger um die Frage gegangen, wer die Schuld für die Schäden trägt, sondern wie die Risiken gesellschaftlich verteilt werden und wer die entstehenden Schäden tragen sollte.« (Perthus 2016: 29)

- Die dritte Linie am Ende des 20. Jahrhunderts zeichnet sich durch wandelnde und brüchige soziale Strukturen bzw. durch eine »Erosion zentraler Institutionen der >organisierten Moderne`(Wagner 1995) - Arbeit, Familie und Sozialstaat« aus (Bührmann 2012: 145 [Herv. i. O.]). Diese Entwicklungen führen zu einer erneuten Individualisierung sozialer Probleme wie Arbeitslosigkeit, Pflege oder gar Kinderbetreuung, von denen der Staat immer mehr absieht und sie "sowohl finanziell wie auch strukturell in Frage« stellt (Castel 2009: 26). Zygmunt Bauman beschreibt diese Veränderung als einen »Ausverkauf staatlicher Hoheitsrechte im Bereich der Bereitstellung von Sicherheit und Gewißheit. Der Staat als wichtigster (vielleicht sogar monopolistischer) Anbieter von Sicherheit und Gewißheit nimmt die Sorgen und Ansprüche seiner Untertanen nicht mehr ernst. (Bauman 2000: 216) Schließlich änderte sich mit der Betonung des ökonomischen Denkens und der Verschiebung politischer Orientierung »von wohlfahrtstaatlichen Zielsetzungen zu solchen von Wettbewerbsfähigkeit und (ökonomischem) Wachstum « (Virchow 2008: 230 zit.n. Perthus 2016: 30) auch das politische Kalkül des Risikos. Das Risiko wird ab dann »teilweise entsozia- 
lisiert, individualisiert und privatisiert, damit private Individuen eigenverantwortlich für ihren Schutz sorgen.« (Perthus 2016: 30) Das Dispositiv der Solidarität wird so durch das Dispositiv der Sicherheit ersetzt, für die jeder alleine sorgen muss (vgl. ebd.).

Die sich wandelnden Entwicklungen der individuellen Haftung, kollektiven Solidarität und der erneut privatisierten Sicherheit zeigen, wie die diskursive Präsenz des Risikos und seine Formen in unterschiedlichen Kontexten thematisiert wurden. Je nach den wirtschaftlichen Fortschritten und verknüpft mit der Etablierung eines rechtlichen Systems (wie François Ewald im Verweis auf die Erklärung der Menschen- und Bürgerrechte (franz. Déclaration des Droits de l'Homme et du Citoyen) und des Code civil (franz. Code civil des Français) gezeigt hat (vgl. Ewald [1986] 1993: 59ff), wird das Risiko und seine Vorbeugung allmählich zum Gegenstand gesellschaftlicher Abhandlung.

\subsubsection{Globale Dimension des Risikos}

Das Problematisieren des Risikos findet im 20. Jahrhundert auch auf einer breiteren Skala statt. Vor allem die Erfahrungen mit den Atomwaffen in Hiroshima und Nagasaki und später auch die Nuklearkatastrophen in Kernkraftwerken Tschernobyl oder Three Mile Island haben das Potential der Bedrohung auf eine globale Ebene erweitert. Die Globalisierungs- und Digitalisierungsentwicklungen haben die globale Dimension des Risikos gezeigt und so zu einer erneuten Reflexion der bestehenden und möglichen Risiken und Gefährdungen gezwungen. In diesem Zusammenhang hat Anthony Giddens sieben Aspekte eines möglichen Risikoprofils der modernen, globalen Gesellschaft herausgearbeitet und folgenderweise skizziert:

- ")Globalisierung von Risiken〈 im Sinne ihrer >Verstärkung‘; Beispiel: der Atomkrieg kann das Überleben der Menschheit bedrohen;

- >Globalisierung von Risiken Ereignisse $<$, die jeden oder zumindest eine gewaltige Anzahl der Bewohner des Planeten betreffen; Beispiel: Veränderungen der globalen Arbeitsteilung;

- Risiken, die aus der >gestalteten Umwelt< bzw. der >vergesellschafteten Natur hervorgehen: das Einsickern menschlichen Wissens in die materielle Umwelt

- die Entstehung >institutionalisierter Risikoumwelten $\prec$, die die Lebenschancen von Millionen berühren; Beispiel: Investitionsmärkte;

- >Bewußtsein vom Risiko als Risiko<: die >Wissenslücken` bei Risiken lassen sich nicht mehr durch religiöses oder magisches Wissen in >Gewißheiten`verwandeln; 
- die >weite Verbreitung des Risikobewußtseins`; viele der Gefahren, denen wir kollektiv gegenüberstehen, sind einer großen Öffentlichkeit bekannt;

- >Bewußtsein von den Grenzen des Expertenwissens‘; kein Expertensystem kann von den Experten restlos beherrscht werden, wenn man die Konsequenzen in Betracht zieht, die sich aus der Übernahme von Fachprinzipien ergeben.« (Giddens [1990] 1995: 156 [Herv. i. O.])

Die ersten vier genannten Risikogruppen können um weitere gegenwärtige Faktoren erweitert werden, wie die globale Erwärmung, transkontinentale und ökologische Migration, Cyberterrorismus usw. Die letzten drei Aspekte des Risikoprofils globaler Gesellschaften hängen mit der Unmöglichkeit einer transparenten massenmedialen Berichterstattung zusammen, was bspw. bei der Brexit-Kampagne, der US-Wahlen 2017 und 2020 oder anhand der populären Desinformationsportale $\mathrm{zu}$ beobachten ist. Gerade die Unkontrollierbarkeit des Wissensflusses stellt neue Risiken dar, die von den Regierungen nicht selten als Grund für verstärkte Überwachung und Zensur dienen. Auf den Grundlagen eines solchen globalen Risikokonzepts kann dann versucht werden, »bevölkerungs- bzw. populationenbezogene oder individuumzentrierte Prävention zu praktizieren. Dabei darf man sich nicht täuschen lassen: es kann sein, daß das Risikokonzept explizit gar nicht genannt, sehr wohl aber seine spezifische Methodik realisiert wird. Insofern kann man bereits von seiner Verallgemeinerung sprechen, die in Alltagshandeln eingeht.« (Schulz \& Wambach 1983: 9)

Hier zeigt sich die spezifische Seite des Übergangs vom Risiko zur Prävention, die sich in konkreten Regierungspraktiken realisieren kann. Paradox bleibt dabei, dass die Risiken, wie Anthony Giddens betont, »notwendig sirreak [sind], denn ein deutliches Bild von ihnen könnten wir uns nur machen, wenn sich Ereignisse abspielten, die zu schrecklich sind, um sie sich auszumalen." (Giddens [1990] 1995: 167) Die betäubende Wirkung solcher Risiken besteht darin, dass die breite Bevölkerung einfach lernt, mit ihnen klarzukommen. Sie sind anwesend, doch die Vorstellung ihrer Bedrohung kann einerseits nicht überprüft werden, andererseits wirkt sie ziemlich paralytisch und verunmöglicht eine normale Lebensweise, was am Ende zur Entwicklung verschiedener Anpassungsreaktionen führt (vgl. a.a.O.: 168).

\subsubsection{Vom Risikodiskurs zu Präventionspraktiken}

Die Profilierung des Risikodiskurses fängt einerseits mit seinen historischen Grundlagen an, andererseits mit der Erzeugung einer bestimmten Empfindlichkeit gegenüber spezifischen Situationen, Prozessen oder Eventualitäten. Die Begrifflichkeiten wie Risiko, Gefahr, Verletzlichkeit, Ungewissheit, Unsicherheit, Unbestimmtheit oder Kontrollverlust antizipieren eine quasi entstandene Lücke, 
die mit dem Wissen um das Nicht-Wissen, also um etwas, was noch nicht existiert, aber mit einem mehr oder weniger sicheren Wahrscheinlichkeitsgrad auftreten kann, gefüllt werden muss, damit sie intelligibel und regierbar gemacht werden kann. Es ist diese Wissens-Lücke, die Sorgen und Unbehagen schafft und damit Regierungspotentiale erzeugt. Das Empfindlich-Machen für bestimmte Risiken kann dann auf das Kontrollieren und Regieren ganzer sozialer Schichten und »ihrer >vorausschauenden Verwaltung aus der Distanz«« zielen (Castel 2011: 27 [Herv. i. O.]). So können gewisse Individuen oder Bevölkerungsgruppen als risikoreich eingestuft werden, womit die Einführung entsprechender Mechanismen ihrer Kontrolle und Überwachung einhergeht. ${ }^{66}$

Ähnlich verhält sich der Risikodiskurs bei der Sorge um die Gesundheit der Bevölkerung und des Einzelnen, indem anstelle einer diagnostizierten Krankheit »die potentielle Krankheit als statistisch virtuelles, als prognostizierbares, kalkulierbares und eventuell vermeidbares Faktum [tritt]. Dieses Schema beherrscht unterdessen viele Interventionsbereiche. Ob somatische, psychische, soziale $>$ Fehlentwicklungen auftauchen, es wird versucht, sie so früh wie möglich zu erkennen und im Keime zu ersticken." (Schulz \& Wambach 1983: 9 [Herv. i. O.]) Sodann verbinden sich der Risikodiskurs mit der Kriminalisierung einzelner Bevölkerungsgruppen, die durch Fehlentwicklung für die Gesellschaft bedrohlich sein können:

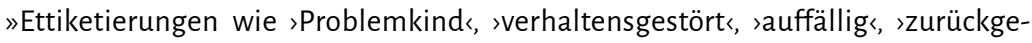
blieben<, >Schulversager<, >Schulschwänzer<, >Ausreißer etc. sollen auf diejenigen Kinder und Jugendlichen aufmerksam machen, die aufgrund ihrer Verhaltensweisen auf dem Wege zu ernsthafteren Vergehen und Verstößen sind. Zunächst werden die Symptome dieser >Prädelinquenz` anhand von individuellen Verhaltensweisen in Kindergarten, Vorschule, Schule, Familie aufgespürt, wobei, wie gesagt, immer davon ausgegangen wird, daß, wenn nicht rechtzeitig interveniert wird, aller Wahrscheinlichkeit nach die Eskalation zu kriminellen Handlungen eintritt. Eine direkte Wechselbeziehung zwischen gegenwärtigen Verhaltensauffälligkeiten und künftiger Kriminalität wird aufgezeigt: z.B. zwischen Leseschwäche und Delinquenz (Taglianetti 1975; de Hirsch 1972), ja, sogar zwischen Leseschwierigkeiten und Neigung zu Cewalttätigkeit (Andrew 1979), zwischen Sprachstörungen und Delinquenz (Mulligan 1972; Stevenson u.a. 1978) oder insgesamt zwischen Schulversagen bzw. Drop-out und Delinquenz (Elliot $\&$ Voss 1974). Die Korrelation zwischen individuellem Risikoverhalten und Kriminalität als statistische Größe scheint desto beliebiger zu sein, je jünger der Symptomträger ist.« (Hellerich \& Wambach 1983: 130 [Herv. i. O.])

66 Diese Identifikation von Risikogruppen und -Individuen könnte sich dabei nach Robert Castel quantifizierbarer Methoden bedienen, die jeder Cruppe und jedem Einzelnen sein eigenes Risikoprofil erstellen kann: »An die Stelle von Menschen aus Fleisch und Blut tritt das Cewölk statistischer Korrelationen.« (Castel 2011: 27) 
Somit konnte eine wissensbasierte Problematisierung des Risikos aus der Prävention ein Regierungsinstrument machen, das auf vielen Gebieten Anwendung findet: »Getreu der alten Volksweisheit >Vorbeugen ist besser als Heilen wird der Präventionsgedanke in Medizin und Gesundheitswesen, bei der Unfallbekämpfung, in der Äußeren Sicherheit und auch im Bereich der Inneren Sicherheit und Kriminalitätsbekämpfung aufgegriffen (Frevel \& John 2014:347 [Herv. i. O.]). ${ }^{67}$ Umgekehrt haben auch Methoden aus anderen Gebieten Eingang in die Präventionsprogramme gefunden, wie das Beispiel des Screenings veranschaulicht, das aus polizeilichen bzw. polizeiförmigen Fahndungsmethoden übernommen wurde (vgl. Schulz \& Wambach 1983: 9). Gerade mit der Entwicklung und Etablierung wissenschaftlicher Forschung konnte ein qualitativer Bruch in der Betrachtung der Prävention erkennbar werden.

Der gängige Blick auf die Prävention entziffert diese als Einführung von Maßnahmen, die der Entstehung einer unerwarteten oder bedrohlichen Situation vorbeugen sollen. Prävention oder Vorbeugung suggerieren zuerst ein reflexives Schutzbedürfnis, »wird doch impliziert, dass die Vermeidung (Prophylaxe) bzw. die Verhinderung (Prävention) schlechter Dinge (Erkrankung, Verletzung, Viktimisierung etc.) doch >gut sei.« (Frevel \& John 2014: 347 [Herv. i. O.]) Außerdem sind Vorsorge und Prävention seit den ersten Kulturen bekannt und also historisch und erfahrungsgemäß begründet: "Schäden durch verschiedene Maßnahmen zuvorkommen, als Bedeutung des lateinischen >praevenire<, wie zum Beispiel Schutz gegen Angriffe oder das Anlegen von Nahrungsvorräten, hat es bereits in Hortikulturgesellschaften als Vorsorgetechniken für die erntelose Zeit und zur Vorbereitung auf die nächste Aussaat gegeben (vgl. Schülein 1983: 23).« (Perthus 2016: 27 [Herv. i. O.]) Doch erst das Umwandeln von Unsicherheiten in wissenschaftlich generierte Gewissheiten (vgl. Bröckling 2008: 40) hat aus den präventiven Maßnahmen Selbstverständlichkeiten und notwendige Bestandteile der Politik gemacht. Die »Generalisierung des Risikos« (Schulz \& Wambach 1983: 9) sorgte für Akzeptanz präventiver Interventionen, die die ganze Gesellschaft als »risikobehaftet begriffen und damit zum Objekt von präventiven Strategien « (Perthus 2016: 32) machten. Ihre Auswirkungen treten dann nicht nur in Form von Gesetzen oder Handlungsempfehlungen auf, sondern wirken gleichzeitig auch

67 Bernhard Frevel und Tobias John greifen diesen Gedanken auf und beziehen sich in ihren Analysen zur kooperativen Sicherheitspolitik auf das Stufenkonzept der Prävention von Cerald Caplan (1964), dessen primäre Prävention »auf die gesellschaftlichen Bedingungen von hier: abweichendem/kriminellem - Verhalten [zielt].« (Frevel \& John 2014: 347) Gerade die Produktion und die Differenzierung des kriminellen Verhaltens bildeten den Ausgangspunkt für Foucaults Untersuchungen zu Einsperrungs- und Überwachungspraktiken. Die Kategorien der Unterteilung in delinquente und konforme Verhaltensweisen ließen sich demnach als diskursiv erzeugte und an Machttechnologien eingebundene Regierungsrationalitäten begreifen (vgl. Foucault [1975] 2008). 
in den Alltagsdiskursen. Eine bedeutende Rolle spielt dabei das Übertragen des Risikos auf die Zukunft. So müssen die Subjekte schon heute kalkulieren, wie sie ihre künftigen Arbeits-, Familien- und Lebenslagen sichern können.

Die gleichzeitige Globalisierung und Individualisierung des Risikos geht bei der Bevölkerung wie bei den Einzelnen mit dem »Versprechen auf Sicherheit« (Beck [1986] 2016: 25; vgl. auch Castel 2011: 24f) und dem Verlangen nach Schutz und Gefahrenvorbeugung einher und bildet auf dieser Basis - der Verbindung von Selbstund Fremdtechnologien des Regierens - ein präventives Regierungsensemble, welches im Zuge der Problematisierung von Risiko und Sicherheit »Führungsverhältnisse arrangiert « (Opitz 2008: 217) hat, die Michel Foucault mit dem Begriff der Gouvernementalität bezeichnete. Die Gouvernementalität der Gegenwart operiert mit dem etablierten Risikodiskurs und der damit verbundenen Entwicklung des Sicherheitsdispositivs, die im nächsten Teil diskutiert wird.

\subsection{Sicherheit und Gefahr}

Die Sicherheit gehört mittlerweile zu einem unabdingbaren Bestandteil modernder westlicher Gesellschaften. Kaum eine Regierung kann sich den Sicherheitsfragen entziehen, mehr noch, keine Regierung kann sich als legitim darstellen, ohne die Sicherheit des Staates und der Gesellschaft zu garantieren. Sicherheit bietet also eine ideale Schablone, vor deren Hintergrund die Problematisierung des Regierens diskutiert werden kann. Im Folgenden werden daher zuerst drei Achsen des Sicherheitsdiskurses betrachtet, zweitens, die Konturen eines Sicherheitsdispositivs als einer modernen Regierungstechnologie umrissen, und schließlich drittens die Wechselbeziehungen zwischen dem Sicherheits- und dem Präventionsdispositiv beschrieben.

\subsubsection{Die Sorge um die Sicherheit}

Die internationale Beunruhigung hat in den letzten Jahren und Dekaden zugenommen. Zum einen hat sich das zwar bedrohliche, doch immerhin stabile bipolare System mit zwei Weltmächten mit Ende des Kalten Krieges aufgelöst. Die Hegemonie der Vereinigten Staaten als einzig gebliebenen Weltmacht konnte seitdem einen globalen Frieden nicht garantieren. Zu der wachsenden Zahl lokaler Konflikte, in die mittlerweile keine Großmacht eingreifen kann, ohne in eine internationale Isolation getrieben zu werden, kommt auch die wachsende Bedrohung durch den internationalen Terrorismus, vor allem seit der Millenniumswende. Auf dem Feld internationaler Politik steigen daher die Spannungen zwischen den Nationalstaaten, was für eine erhöhte Sensibilität gegenüber der globalen Sicherheit sorgt.

Die Sorge um die Sicherheit findet allerdings auch auf der lokalen bzw. regionalen Ebene statt. Innerhalb nationaler Staaten erregen die Sicherheitsfragen zusammen mit dem Phänomen des Populismus (vgl. Flecker \& Krenn 2009: 329) 
immer breitere Massen und führen nicht selten zu irrationalen und panischen Reaktionen, wie dies die europäischen und nordamerikanischen Flüchtlingskrisen beispielhaft nahegelegt haben. Zusätzlich dazu erfordern die unkontrollierbaren Cyber- und Finanzattacken neue Maßnahmen, die die sensiblen Daten vor Missbrauch schützen sollten. Durch die Digitalisierung und Vernetzung haben globale Sicherheitsfragen zusätzlich an Relevanz gewonnen.

Die einfache Skala der globalen und lokalen Sicherheitsproblematik bietet nur eine Veranschaulichung dessen, wie verbreitet und immanent die Sicherheit als tagtägliches Thema wirkt, abgesehen von Sicherheitsbelangen, die jeden Einzelnen auf unterschiedliche Weise noch konkreter betreffen. Doch der Diskurs um Sicherheit, seine Genealogie und seine gesellschaftliche Funktion lassen sich viel konkreter entlang von drei Achsen diskutieren, die an Michel Foucaults Untersuchungen anknüpfen (vgl. Foucault [1978] 2006).

\subsubsection{Drei Achsen des Sicherheitsdiskurses}

Als erste Achse ist die Raumachse zu nennen. Besonders anschaulich wird dies am Beispiel der Europäischen Union: »Blickt man zurück, so gab es noch nie in der Geschichte Europas so lange Frieden am Stück, jetzt bereits seit 70 Jahren. Und das in einer Welt, in der rings um uns herum über 40 bewaffnete Konflikte schwelen, die jedes Jahr mindestens 170.000 Menschenleben fordern.« (EU 2019) Für die Staaten Europas spielt die Sicherung des Friedens auf dem eigenen Territorium eine andauernd bedeutende Rolle. Und das nicht nur in den modernen Zeiten. Die Geschichte des Abendlandes wird von Sicherheits- und Sicherungsfragen von Anbeginn an durchdrungen, worauf, z.B. die Beschreibungen unbekannter Territorien auf den Europäischen Landkarten hinweisen:

»auf alten Landkarten symbolisierten bedrohliche Tier- oder Fabelwesen (hic sunt leones, hic sunt dragones) unbekannte und daher unkontrollierte Cebiete; aber auch die Bezeichnung von nicht im westlich-europäischen Sinne regierten Völkern als >Barbaren oder >Wilde< verweist auf die Sicherheits- beziehungsweise Unsicherheits- und Bedrohungsvorstellungen, die mit angeblich unregierten Räumen verbunden sind. «(Conze 2018: 128f [Herv. i. O.])

Damit hat, zumindest in Europa, eine Versicherheitlichung unregierter Räume (vgl. ebd.) begonnen, die das Territorium als Machtsymbol erschlossen und problematisiert hat. Die Territorien und Räume besitzen unterschiedliche Qualität, werden von mehr oder weniger bedeutenden Transport- und Handelswegen durchkreuzt und müssen also nicht nur gesichert werden, sondern sichern die Stabilität des Staates selbst. Räume müssen deshalb erst dann versichert werden, wenn sie einem spezifischen Zweck dienen sollen, nämlich dem Machterhalt und dem RegierbarMachen. Diese souveräne Ausübung der Macht richtet sich demnach »auf die Grenzen eines Territoriums.« (Foucault [1978] 2006: 27) 
Als eine zweite Achse des Sicherheitsdiskurses lässt sich die Achse der Bevölkerung nennen. Das Territorium regierbar zu machen heißt, seine Bevölkerung durch disziplinarische Techniken zu Gehorsam und Unterwerfung zu führen. Eine solche disziplinarische Sicherheitstechnologie, die nicht über unmittelbaren Zwang, sondern durch Errichten von Praktiken der Selbstführung agiert, lässt sich an der Geschichte des HIV/AIDS-Diskurses ablesen:

»Von seinen Anfängen bis zu seiner aktuellen pandemischen Verbreitung steht HIV/AIDS für ein komplexes Beziehungsgefüge zwischen Regierenden und Regierten, auf dem sich ein mächtiges transnationales Schutz- und Sicherheitsdispositiv errichten konnte. Mit einer Konsequenz wie kein anderes Dispositiv vor ihm hat das im sepidemischen Zeitalter (Singer 1993) von AIDS entstehende Sexualitätsdispositiv den Sex bestimmter Bevölkerungsgruppen mit Ansteckung und Tod identifiziert und den Topos der bedrohlichen Sexualität aktualisiert, um scharfe Grenzen zwischen sgefährdeten und sgefährlichen Körpern zu ziehen. Die epidemiologische Konstruktion von >Risikogruppen<- im Gegensatz zu riskanten >Praktiken <- ist von Beginn an eine wirkmächtige Aussageformation im AIDS-Diskurs. Sie stilisiert in den USA der 1980er Jahre Homosexuelle, Schwarze und Lateinamerikaner_innen zu Agent_innen des Todes, so wie sie heute ganze Kontinente und Regionen - von Afrika über die karibischen Inseln bis Lateinamerika und weiter nach Südostasien - als Cefahrenzonen entwirft.« (Laufenberg 2014: 325 [Herv. i. O.])

Am Beispiel des Sexualitätsdispositivs wird deutlich, wie die Bevölkerungsgruppen differenziert, normiert, kriminalisiert und eben dadurch regierbar gemacht werden. Das Differenzieren anhand von Geschlechterdefiziten und Konstruktionen von Risiken etabliert »eine Abhängigkeitsbeziehung zwischen Instanzen der Sicherheit und Ge- bzw. Versicherten [...]. Durch diese Abhängigkeit stehen die Versicherten in der Schuld bzw. in der Macht der Schutzgewalten. Sie sind durch diese Macht in einem eminenten Sinne verwund- und ausbeutbar. Darum ist Sicherheit gefährlich." (Folkers 2018: 464) So wird die Bevölkerung durch statistische Verfahren, wirtschaftliche Zielsetzungen und wissenschaftliche Problematisierungen nach und nach zum Regierungsgegenstand und Objekt der Machttechniken gemacht (vgl. Foucault [1978] 2006: 120).

Eine dritte Achse des Sicherheitsdiskurses entfaltet sich vor dem Hintergrund des Zeithorizonts. Das Sicherheitsversprechen richtet sich nicht nur auf Räumlichkeiten und auf die Bevölkerung und deren Bestandteile, sondern auch auf die »Überwindung der Unbestimmtheit der Zukunft. (Kaufmann 2015: 26) Das hängt mit der wachsenden Risikosensibilität zusammen, die es ermöglicht, die Dimensionen der Sicherheit zu erweitern und zeitlich unbegrenzt zu halten. Risiken als Gefahren, die noch nicht entstanden sind, aber in der Zukunft entstehen können, erzeugen Unsicherheit: »Unsicherheit (insecurity) wird hier als Ungewissheit 
(uncertainty) gedeutet, dadurch entkonkretisiert und zum Gegenstand nicht mehr reaktiver, sondern >proaktiver Sicherheitspolitik« (Christopher Daase) gemacht.« (Conze 2018: 55) Damit wandelt sich das Verständnis der Sicherheit als reaktiver Politik zur Sicherheit als proaktiver bzw. vorbeugender Politik. Mit ihr entstehen auch weichere Formen der Sicherheitsmaßnahmen, die sich zweckrationaler Strategien bedienen und die

»in ihrem Kern und Selbstverständnis auf ein subjekt- und situationsunabhängiges Durchspielen von Unsicherheitssituationen hinaus[laufen]. Subjekt- und situationsunabhängig (und damit dekontextualisiert) ist dieses Durchspielen, weil es unabhängig vom konkreten Fall einem bestimmten Kalkül gehorcht. Gemeint ist Wahrscheinlichkeitskalkül, das vor der Renaissance unbekannt war, im 17./18. Jahrhundert einen rasanten Aufstieg erlebte und sukzessive soweit universalisiert wurde, dass der Wahrscheinlichkeitsbegriff im 20. Jahrhundert >den Begriff der Kausalität allmählich verdrängt hatı.« (Bonß 2010: 44 [Herv. i. O.])

Basierend auf dem Wahrscheinlichkeitsgrad des Auftretens einer Gefahr können auch Sicherheitsfragen gradiert und grenzenlos werden. Was als Sicherheitsrisiko gilt und was nicht mehr, kann auf einer Skala eingestuft und dementsprechend als politische Agenda verzeichnet werden. Dies bezeugt der Begriff der erweiterten Sicherheit, welcher "seit den 1970er Jahren - und das nicht nur in Deutschland aus dem politischen Vokabular nicht mehr verschwunden [ist].« (Conze 2018: 50) So können dank der proaktiven Sicherheit, d.h. zwecks Vermeidung bzw. Minimierung des Risikos, notwendige Schutzmaßnahmen veranlasst werden, die die Aktualität der Gefahr, die bekämpft und abgewehrt werden soll, immer wachhalten (vgl. Demirović 2008a: 245).

Die drei schematischen Achsen des Sicherheitsdiskurses veranschaulichen, wie sich die Rationalität der Sicherheit sukzessive entwickelt hat und sich Räume, Bevölkerung und Zukunft als Elemente der Sicherheitspraxis vereinnahmt hat. Die Verbindung dieser Elemente zusammen mit einer bestimmten Rationalisierung des Politischen (vgl. Opitz 2008: 218), die das Regieren dieser Elemente intelligibel macht, hat die Entwicklung eines Sicherheitsdispositivs ermöglicht, dessen Aufgabe darin besteht,

»[d]as Territorium nicht mehr befestigen und markieren, sondern die Zirkulationen gewähren lassen, die Zirkulationen kontrollieren, die guten und die schlechten aussortieren, bewirken, daß all dies stets in Bewegung bleibt, sich ohne Unterlaß umstellt, fortwährend von einem Punkt zum nächsten gelangt, doch auf eine solche Weise, daß die dieser Zirkulation inhärenten Gefahren aufgehoben werden.« (Foucault [1978] 2006: 101) 


\subsubsection{Konturen des Sicherheitsdispositivs}

Das Sicherheitsdispositiv beschreibt zunächst eine analytische Kategorie, mit der die Regierungsarrangements der Moderne, die versuchen, ein kontrollierbares Territorium, disziplinierte Individuen und eine gegen Gefahren und Prozessstörungen gesicherte Bevölkerung einzurichten (vgl. Foucault [1978] 2006: 27), untersucht werden können. Dieser Regierungsmechanismus lässt sich durch folgende vier Aspekte umschreiben:

Zum einen ist für eine solche Regierungspraxis nicht das Betreiben eines sicheren Überlebens zentral, sondern die Steigerung der wirtschaftlichen Produktivitätsmöglichkeiten, der Natalität und Vitalität der Bevölkerung sowie der Militärkraft. Um dies alles störungsfrei sichern zu können, bedient sich das Sicherheitsdispositiv der beobachteten Gesetzmäßigkeiten des Marktes und der damit beginnenden liberalen Rationalität. Die Entstehung des Sicherheitsdispositivs bzw. der Sicherheitsdispositive - die auf unterschiedlichen Territorien und zu unterschiedlichen Zeiten verschiedene Formen angenommen haben -, ist "an die liberale Gesellschaft, den liberalen Staat und an liberale Gouvernementalität gekoppelt.« (Conze 2018: 103) Gerade der liberale Ordnungsgedanke strukturiert aufs Neue die Machtverhältnisse zwischen seinen Komponenten. Die Macht im (liberalen) Sicherheitsdispositiv operiert dann »nicht normativ und ausgrenzend, sondern lässt die Dinge geschehen. Sie orientiert sich an statistischen Normalitätserwartungen, die die Grenzen des Akzeptablen festlegen, und stützt sich auf ein Kalkül, das die Kosten der Machtanwendung einbezieht.« (Demirović 2008a: 242) Damit liegt das Spezifikum eines Sicherheitsdispositivs darin, »die Verbindung liberaler Freiheit (des Einzelnen) mit einem fortexistierenden Koordinations- und Regulationsinteresse des Staates $\mathrm{zu}$ schaffen (Conze 2018: 103).

Zum anderen agiert das Sicherheitsdispositiv als eine Rationalität, bei der es darum geht, »weder den Standpunkt des Unterbundenen noch den Standpunkt des Verbindlichen einzunehmen, sondern ausreichend Abstand zu gewinnen, damit man den Punkt erfassen kann, an dem die Dinge sich ereignen, seien sie nun wünschenswert oder nicht.« (Foucault [1978] 2006: 75) Dieser analytische Blickwinkel entlarvt das Dispositiv der Sicherheit als eine immanente Regierungsordnung, die die Verläufe nicht erzwingt, sondern sie kanalisiert, die Dinge nicht erzeugt, sondern sie differenziert, die Bevölkerung nicht gefährdet, sondern zum Objekt der Sicherheitsmaßnahmen macht, um »deren Lebensbedingungen zu gestalten und zu sichern. (Folkers 2018: 449) Gerade dank diesem immanenten Bezug zur Realität konnte sich das Sicherheitsdispositiv erfolgreich etablieren. Und zwar indem es unaufhörlich seine Elemente in Bezug zueinander gebracht, in Gang gesetzt und überwacht hat: »das Gesetz verbietet, die Disziplin schreibt vor, und die Sicherheit hat - ohne zu untersagen und ohne vorzuschreiben, wobei sie sich eventuell einiger Instrumente in Richtung Verbot und Vorschrift bedient - die wesentliche 
Funktion, auf eine Realität zu antworten, so daß diese Antwort jene Realität aufhebt, auf die sie antwortet - sie aufhebt und einschränkt oder bremst oder regelt.« (Foucault [1978] 2006: 76)

Außerdem ließe sich das Sicherheitsdispositiv im konkreten historischen Wandel als ein Faktum, als eine faktische Wirksamkeit beschreiben. Ob auf direkte und sichtbare Weise (beobachtbar an architektonischen Arrangements wie Grenzen, Barrieren, Waffensystemen usw.) oder indirekt und unterschwellig (wie im Falle der Überwachungssysteme, Propaganda oder Gesetze) funktioniert das Sicherheitsdispositiv als ein Ensemble ganz konkreter Objekte, Praktiken und Subjektivierungsformen. Damit wird dem Begriff des Dispositivs eine doppelte Form verliehen:

»Es ist nicht nur das, was von einer einheitlichen Logik durchwebt ist, es ist auch das Prinzip, das diese einheitliche Logik weiter ausbreitet, ein wirksames Dispersions- und Distributionsprinzip. Die Vorsilbe des Begriffs wird oft übersehen. Es ist ein Dis-positiv, also weder eine Gegebenheit, die einfach so beobachtbar wäre, noch eine bloße epistemische Konstruktion. Das Dis-positiv ist ein nicht positiver, nicht unmittelbar sichtbarer Teil der Wirklichkeit. Etwas, das die Positivitäten, die Gegebenheiten anordnet, verteilt und verbindet.« (Folkers 2018: 213)

Schließlich nimmt das Dispositiv der Sicherheit außer räumlichen auch zeitliche Konturen an, wie dies bereits bei der Problematisierung des Sicherheitsdiskurses erwähnt wurde (vgl. Conze 2018: 127). Die erzeugten Gefährdungen und Risiken ziehen dann nicht nur das Versprechen von Sicherheit und Ordnung nach sich (vgl. a.a.O.: 147), sondern auch eine zeitliche Vorstellung von dem, wie und vor allem wann die Gefahren abgewehrt werden sollen. Wenn sich Sicherheit in konkrete Maßnahmen umschreibt, so folgen diese einem Zeitplan bzw. einer Zeitachse, auf der sich die jeweiligen Schritte ereignen sollen. Damit eröffnet das Dispositiv die Zeitasche in Richtung Vergangenheit und in Richtung Zukunft: "Die mühevolle Entstehungsgeschichte des Dispositivs bleibt als sein Gedächtnis präsent und spannt so ein Feld geteilter Problemlagen und konditionierter Möglichkeiten auf, das zukünftige Reaktionsbildungen nicht determiniert, aber mehr oder weniger wahrscheinlich macht.«(Folkers 2018: 457f) Dieses Gedächtnis enthält Taktiken und Strategien des Machterhalts, die sich an verschiedene geschichtliche Entwicklungen bzw. Ereignisse kontinuierlich anpassen. So war bspw. für die Regierungen des 17. und 18. Jahrhunderts der Nahrungsmangel »das Musterbeispiel für ein Ereignis, das es aus einer ganzen Anzahl von Gründen zu vermeiden gilt, die offensichtlich sind.« (Foucault [1978] 2006: 53) Andererseits haben die Entwicklungen in den städtischen Räumen zur Bearbeitung ihrer Zukunft geführt, d.h. 
»daß die Stadt nicht im Zusammenhang einer statischen Wahrnehmung aufgefaßt oder gestaltet wird, die für den Augenblick die Vollkommenheit der Funktion gewährleisten würde, sondern sie öffnet sich für eine nicht genau kontrollierte oder kontrollierbare, nicht genau bemessene oder meßbare Zukunft, und die gute Stadtgestaltung ist genau folgendes: dem Rechnung tragen, was geschehen kann.«(a.a.O.: 39)

Mit der Entwicklung des Sicherheitsdispositivs wird also eine permanente Problematisierung der Zukunft in die Regierungspraxis eingeführt (vgl. Conze 2018: 44). Die vier Konturen umrahmen skizzenhaft die Wirkungsweise eines Dispositivs der Sicherheit. Dieses lässt sich nicht als ein bloßes geschichtliches Ereignis begreifen, sondern eher als eine wandelnde Form der Regierungstechnologien, die sich durch die Anpassung an neue Ereignisse und Widerständigkeiten fortentwickeln. So könnte sich im Regierungsdenken der Staatsräson das Disziplinardispositiv als eine entsprechende Regierungstechnologie durchsetzen, im liberalen Regierungsdenken das Sicherheitsdispositiv und, als Fortsetzen dieser Logik, im neoliberalen Regierungsdenken das Präventionsdispositiv.

\subsubsection{Vom Sicherheits- zum Präventionsdispositiv}

Eine Trajektorie des Übergangs vom Dispositiv der Sicherheit zum Dispositiv der Prävention beschreiben zu wollen heißt, diejenigen Wende- und Bruchpunkte aufzugreifen, die auf eine veränderte Wahrnehmung von Regierungsproblemen hinweisen. Auch Gefahr oder Sicherheit werden damit als Ergebnisse »einer Deutung von Realität « (Conze 2018: 70) verstanden, die sich im Zeitverlauf wandeln und unterschiedliche Formen aufnehmen kann. Um diese Wandlungen beobachten zu können, erweisen sich vier Perspektiven als besonders hilfreich.

Die erste, historisch-philosophische Perspektive nimmt den Wandel von der Vormoderne zur Moderne in den Blick und mit ihm auch den Wandel der Logik von Welterklärung. So basierte die vormoderne Welterklärungslogik darauf, »dass alles auf einen absolut geltenden Ursprung zurückgeführt und als seine Hervorbringung verstanden werden konnte. (Vobruba 2010: 252) Demgegenüber machen die Menschen der Moderne die Erfahrung, »dass hinter ihnen nichts ist, sie sehen sich >rückverwiesen auf sich selbst<. Dies hat Konsequenzen. >Diese Rückverwiesenheit auf sich selbst wurde überwiegend als ambivalente Erfahrung zwischen Verlust von Sicherheit und Chance zur Selbstgestaltung registriert und beschrieben.« (ebd. [Herv. i. O.]) Der moderne, auf sich selbst verwiesene Mensch muss seine Lebens- und Überlebenschancen an sich selbst binden und statt sich auf eine vis major zu stützen, muss er in sich selbst die nötigen Ressourcen aufbauen, die eigene Kalküle präzisieren, das Funktionieren der Gesellschaft überblicken und die Selbstgestaltung anhand der vorgeschriebenen Prinzipien praktizieren. Das moderne Individuum muss mithin die Sicherheit zu einem der zentralen Ziele seiner 
Selbstgestaltung machen (vgl. a.a.O.: 253). Dieser von der Vormoderne zur Moderne stattfindende Wandel lässt sich an der Behandlung von Krankheiten und ihrer Prävention gut illustrieren. Als Beispiel lassen sich hier die Sicherheitsmaßnahmen des zentralafrikanischen Stammes Lele zur Bronchitis nennen. Die Prävention gegen Bronchitis besteht bei diesem Stamm nicht darin, »sich auf die Umwelt anders einzustellen oder die Umweltbedingungen zu verändern. Statt sich um sheilklimatische Bedingungen $\mathrm{zu}$ bemühen, kommen vielmehr magische Praktiken zum Einsatz, wie Amulette, die gleichsam präventiv zu tragen sind.« (Bonß 2010: 39) Bei diesem Stamm wird die Sorge um eine höhere Gewalt praktiziert. Demgegenüber präferiert die moderne Behandlung entweder eine Verhaltens- (Einstellung auf die Umwelt) oder Verhältnisprävention (Veränderung der Umweltbedingungen), bei der die Individuen mitmachen müssen. Die Prävention legt daher nicht so sehr den Fokus auf die Gefahren als vielmehr auf die Konzentration auf ihre (eigenen) Behandlungsmöglichkeiten.

Eine zweite, sozial-staatliche Perspektive konzentriert sich auf das Verhandeln der Sicherheitsfragen zwischen dem Individuum und dem Staat. Wie bereits gezeigt wurde, findet am Übergang vom Sicherheits- zum Präventionsdiskurs eine Dynamik statt, "angesichts derer von einer >Wiederkehr der sozialen Unsicherheit< zu sprechen ist.« (Castel 2009: 25 [Herv. i. O.]) Diese Dynamik löst die Debatte aus, inwiefern der Staat die Rückkehr der sozialen Unsicherheit begünstigt und wie die Prozesse der »'Entkollektivierung〈 beziehungsweise der >Re-Individualisierung« (ebd. [Herv. i. O.]) vor dem Hintergrund der sich neu etablierenden Machtregimes $\mathrm{zu}$ verstehen sind. Die Verlagerung der Verantwortung auf die Individuen kann unterschiedlich interpretiert werden, so Matthias Bohlender: "Die einen machen ideologiekritisch den >Neoliberalismus verantwortlich; die anderen sprechen im soziomoralischen Jargon von >Überforderungく und >Maßlosigkeit<; wieder andere bedienen sich einer bio-politischen Sprache und befürchten eine Art >sanften Tod durch >demographischen Wandek.« (Bohlender 2010: 122 [Herv. i. O.]) Hier findet die Prävention ihren Ankerpunkt darin, dass sie einen zukünftigen Risikohorizont erzeugt. Indem die individuellen Belange sehr unterschiedlich ausfallen und nicht mit einer einzigen Regierungsstrategie behandelt werden können, provoziert die Prävention eine Suche nach einem gemeinsamen Nenner, auf den sich ihr Fokus richten kann. Was für sie fehlt ist

»eine neue bedrohliche, gefährliche Figur, auf die man sich einigen und die die institutionelle Regierungsfurcht erregen könnte, um mit neuen Mitteln und Instrumenten diese Figur zu identifizieren und zu vermessen, von allen Seiten einzukreisen, zu sfördern und zu fordern<, zu ermächtigen, zu überwachen und zu disziplinieren. Was fehlt, ist der fokussierte Zielpunkt einer bislang noch zerstreuten Angst (vor Prekarität, Terrorismus, Migration und befremdlicher Religiosität), der die Akzeptabilitätsbedingungen für die neuen Regierungstechnologien her- 
vorbringen könnte. Einstweilen begnügt man sich noch mit dem >Arbeitslosen «.« (Bohlender 2010: 122 [Herv. i. O.])

Eine dritte, epistemisch-theoretische Perspektive zeigt die Verschiebung von der Sicherheit als einer natürlich gegebenen Konstante zur Sicherheit als einer kontextuell und diskursiv hervorbrachten Kategorie. Die Wissenschaft hat sich mithin davon verabschiedet, "aus wissenschaftlicher Warte vorab festlegen $\mathrm{zu}$ wollen, was Sicherheit >ist< und welches Problem >wirklich Sicherheit wird nicht als kontextunabhängige, objektive Gegebenheit angesehen, sondern als eine diskursive Figur, deren kontextspezifische soziale Verwendung Probleme erst in Sicherheitsprobleme transformiert und sie einer spezifischen Behandlung zugänglich macht.«(Opitz 2008: 214f [Herv. i. O.]) Die sodann vollzogenen Akte der Securitization - der Hervorhebung ausgewählter Themen und Rechtfertigung der dazu notwendigen Gegenmaßnahmen - bauen auf eine vorherige Problematisierung und Politisierung des Risikos und der Sicherheit auf. Das Sicherheitsdispositiv kann daher im gewissen Sinne als Vorläufer des Präventionsdispositivs thematisiert werden, indem es geschafft hat, eine Rationalität des Sicherheitsbewusstseins zu erzeugen und gesellschaftlich akzeptabel zu machen. Es ist diese Art der »Rationalisierung des Politischen« (a.a.O.: 218), die die präventiven Angriffe und Sicherungsmaßnahmen »erst intelligibel macht« (ebd.) und die die Wahrheitsregime erzeugen kann, welche »in die Konstituierung der gefährlichen Subjekte unserer Gegenwart involviert sind.« (Ebd.) Und von gefährlichen zu präventierbaren Subjekten bleibt dann nur ein kleiner Schritt.

Eine letzte emotions-geschichtliche Perspektive kann auch als paradigmatisch für den Übergang und die gleichzeitige Überschneidung von Sicherheits- und Präventionsdispositiv gelten. Gefahr wahrzunehmen und nach Sicherheit zu verlangen sind auch emotionale Reaktionen. Angst und Sorge, Ohnmacht und Schutzbedürfnis, Unsicherheit und Vertrauen begleiten den Sicherheits- und Präventionsdiskurs und überlappen sich dort, wo es um ihre politische Regulierung geht. Werden die Sicherheitsfragen als Schutz gegenüber exogenen bzw. unkontrollierbaren Faktoren dargestellt, kann insbesondere das Vertrauen die Funktion erfüllen, wie Eckart Conze im Verweis auf Niklas Luhmann zeigt, die soziale Komplexität zu reduzieren, um ein sog. Systemvertrauen wiederherzustellen (vgl. Conze 2018: 165). Dadurch kann die Macht gezielter und effektiver eingreifen und wirken. Werden dahingegen die Sicherheitsfragen als endogene, der Gesellschaft und dem Individuum innewohnende Faktoren präsentiert, so bewirkt dies, wie Andreas Folkers im Verweis auf Roberto Esposito nahelegt, immunologische Reaktionsmuster:

»Gefährdungen regen nämlich zu Selbstbeschreibungen an, so wie das Immunsystem eines Organismus immer dann, wenn es durch eine schädliche Beeinträchtigung provoziert wird, eine Selbstrepräsentation erzeugen muss, die es erlaubt, das Eigene vom Eindringenden, das Harmlose vom Gefährlichen zu unterschei- 
den. Die Selbstbeschreibung im Angesicht der Gefahr hat insofern bereits eine sichernde, selbstvergewissernde Funktion.«(Folkers 2018: 215)

In diesem Sinne bezieht sich die provozierte und vorgenommene Beschreibung der Selbstgefährdung auch auf eine allgemeine Gesellschaftsbeschreibung, »die diese Gefährdung in einem größeren Zusammenhang verortet und ihr so einen genuin >sozialen`Sinn gibt.« (ebd. [Herv. i. O.]) Daraus lässt sich schließen, dass einem jeden Dispositiv »eine spezifische Version der Gesellschaft « zugeordnet wird (vgl. a.a.O.: 216), allerdings auch umgekehrt, dass jede Gesellschafts(-selbst-)beschreibung ein bestimmtes Dispositiv ins Leben ruft. Im Fall der neoliberal geprägten Gesellschaftsbeschreibung wächst insbesondere die Sorge um das Emotionale der Individuen (vgl. Penz \& Sauer 2016), die die Sorge um das Vernünftige der Individuen verdrängt:

»Objektivität, Allgemeinheit und auch Beständigkeit zeichnen die Rationalität aus. So ist sie der Emotionalität entgegengesetzt, die subjektiv, situativ und volatil ist. Emotionen entstehen vor allem beim Wechsel der Zustände, bei Veränderungen der Wahrnehmung. Rationalität geht dagegen mit Dauer, Konstanz und Regelmäßigkeit einher. Sie bevorzugt stabile Verhältnisse. Die neoliberale Ökonomie, die zur Steigerung der Produktivität immer mehr Kontinuität abbaut und mehr Unbeständigkeit einbaut, treibt die Emotionalisierung des Produktionsprozesses voran. Auch die Beschleunigung der Kommunikation begünstigt deren Emotionalisierung, denn die Rationalität ist >langsamer «als die Emotionalität. Sie ist gleichsam >ohne Geschwindigkeit‘. So führt der Beschleunigungsdruck zu einer >Diktatur der Emotion<.«(Han 2015: 64f [Herv. i. O.])

Die Rationalität des Präventionsdispositivs baut im Vergleich zur Rationalität des Sicherheitsdispositivs auf die Sorge um die Emotionen, um die Zukunftsängste, um das Noch-Nicht-Geschehene, nicht auf das klar als bedrohlich zu Bezeichnende. Präventiert werden müssen Zukunftsängste, die dem momentanen Sicherheitsgefühl im Wege stehen. Mit anderen Worten, sicher fühlen kann man sich dann, wenn man die Unsicherheiten präventiv durchdenkt. Sicherheits- und Präventionsdispositiv befinden sich daher weder in Kollision noch im Widerstand zueinander. Vielmehr leihen sie sich die Instrumente und Technologien, mit denen das Regieren reibungslos gelingen kann.

\subsection{Zwischenfazit}

Wie eingangs erwähnt, besteht die Aufgabe der gesellschaftstheoretischen Kontextualisierung des Präventionsdispositivs darin, auf drei konkrete Fragenstellungen einzugehen. Und zwar zu untersuchen, welche Veränderungen des Subjektmodus in dem untersuchten Dispositiv zu beobachten sind, welche soziale Wandlungen 
das Entstehen des Dispositivs eingeleitet und begleitet haben und welche MachtWissens-Nexus ihm innewohnend sind.

Bezogen auf die erste Frage lässt sich eine bestimmte Veränderung des Subjektmodus beobachten. So hat sich in der zweiten Hälfte des letzten Jahrhunderts eine Subjektivierung der präventiven Verantwortung vollzogen, in der die individuellen Akteure bzw. »das 'präventive Selbst‘, jenes rationale, krankheitsminimierend agierende Subjekt « (Lengwiler \& Madarász 2010: 16 [Herv. i. O.]) ist, das selbst für seine Gesundheit die Verantwortung trägt. Dagegen konstituiert die individuelle Verweigerung der Prävention »einen zusätzlichen und neuen moralischen Schuldvorwurf.« (Cremer-Schäfer 2016: 20) Dies ist durch einen doppelten Charakter der neuen Subjektform zu erklären:

- Zum einen wird »das selbstständige, mündige Subjekt« (Schulz \& Wambach 1983: 9) gefordert, das zur "Selbstbestimmung und Eigenverantwortlichkeit« (ebd.) fähig ist und den neoliberalen Leitmotiven der »Autonomie<, >Selbstorganisation « und >Zielstrebigkeit« folgt (Bohn 2017: 110 [Herv. i. O.]).

- Zum anderen wird allerdings eben dieses Subjekt »durch die permanente Supervision des Staates und der Unternehmen, gleichgültig, ob diese nun als Hilfe oder Herrschaft geriert, an seiner Entfaltung gehindert und damit negiert « (Schulz \& Wambach 1983: 9) und soll sich, um seiner Gesundheit willen, auf sich selbst beziehen und sich »in der >Achtsamkeit«, >Selbstakzeptanzく und `Selbstwertschätzung« üben (Bohn 2017: 110 [Herv. i. O.]).

Dieser Uneindeutigkeit des Subjekts ist zum einen der »Pluralisierung der Subjektgrenzen« (Beck/Nonß/Lau 2001: 43 zit.n. Poferl 2009: 239) zu verdanken, in welcher »das Individuum (...) als >Quasi-Subjekt< (...) Resultat >und Produzent seiner Vernetzung, Situierung, Verortung, Gestalt ist.« (Beck/Nonß/Lau 2001: 44 zit.n. Poferl 2009: 239 [Herv. i. O.]) Damit erscheint das präventive Subjekt als die größte Gefährdung für sich selbst und gleichzeitig als die alleinige Chance, dieser Gefährdung vorzubeugen. Mehr noch, in Verbindung mit dem Appell an Authentizität, durch den man das, "was man tut, so darstellt, als wäre es voll und ganz Ausdruck des eigenen Selbst (und das noch auch glaubt)« (Schneider 2009: 284) wird das neue, präventive Subjekt als ein freies Subjekt gedacht, als eines, »das alles das, was es soll, von sich aus ohnehin schon will.« (Wernstedt 2017: 194) Hier verbinden sich also zwei Anforderungen, die im Präventionsdispositiv wirken: die Anforderung, das eigene Verhalten als Wahrheit über sich selbst darzustellen, und die Anforderung, das eigene Verhalten als freie Willensentscheidung erscheinen zu lassen. Dieser stumme Zwang zur authentischen Authentizität einerseits und gewollten Freiheit andererseits »ist das strategische Funktionsprinzip der gesellschaftlichen Formierung von individualisierter Subjektivität im Zuge forcierter ssubjektivierender 
Individualisierung« (Schneider 2009: 284 [Herv. i. O.]), die das Präventionsdispositiv begünstigt.

Die Frage der sozialen Wandlungen zeigt, dass das Präventionsdispositiv durch eine veränderte Wahrnehmung des Risikos und des Gefahrensinns entstehen konnte. Zum einen war die Sensibilisierung für Risiken ein Grund »für die Umstellung von Nachsorge auf Prävention. Man will alles unternehmen, damit es erst gar nicht zu Unfällen kommt. (Münkler/Bohlender/Meurer 2010: 8) Dem haben auch die nuklearen Bedrohungen des kalten Krieges stark verholfen und dafür gesorgt, dass sich die Militärdoktrin der Prävention "gewissermaßen auf die gesamte Gesellschaft ausgedehnt [hat].«(Ebd.) Zum anderen wurde mit dem Begriff des Gefahrensinns eine »Verknüpfung von Zukunft und Gegenwart, das Hineinwirken der Zukunft in die Gegenwart« geschaffen (Conze 2018: 119). Das Projizieren zukünftiger Ereignisse in die Gegenwart und der Versuch ihrer Kalkulation führt dann zu ihrer politischen Bearbeitung (vgl. ebd.), zur Entwicklung notwendiger und bestmöglicher Maßnahmen, die auf eine von den zwei Zukünften - entweder der ohne Prävention oder der mit Prävention bzw. auf beide möglichen Zukünfte zugleich - vorbereiten sollen: »Dies alles ist noch kurioser, wenn man mitbedenkt, dass keiner Zukunft und keiner Vergangenheit irgendeine Form der Realität unterstellt werden kann. Die Zukunft `ist nicht wirklich. Sie bietet, wie man auch sagen könnte, keinen Widerstand, sie ist nicht widerständig.« (Fuchs 2008: 364 [Herv. i. O.]; vgl. auch Hölscher 2017) Damit schafft das Präventionsdispositiv die Illusion einer mehr oder weniger gefährdenden Zukunft, die gebändigt und kontrolliert werden muss, damit sie keinen Widerstand leistet, obwohl sie ihn in der Tat auch nicht leisten kann. So gesehen wird Prävention und der darin wirkende Gefahrensinn »zur Regierungstechnik im Sinne von Foucaults Gouvernementalität.« (Conze 2018: 119) Als Regierungstechnologie gelingt es der Prävention, »diese paradox anmutende Zeitform als >natürliche< Voraussetzung des Vollzugs ihrer Operationen und ihrer Selbstreflexion zu behandeln und die offenkundige Künstlichkeit ihrer Temporalität auszublenden. Sie nimmt sich als Realität.« (Fuchs 2008: 364f) Gerade darin, in der inszenierten Selbstverständlichkeit, besteht ihre eigene Gefährdung. Die Taktik, die sie anwendet, beruht auf dem durch das Sicherheitsdispositiv eingeführten und bis in die Körper und Psychen der Individuen eingeflossenen Gefahrensinn: »In einer Welt existentieller Unverwundbarkeit bedürfte es der Vorbeugung nicht.« (a.a.O.: 373f) Die Körper und Psychen der Burnout-Betroffenen wurden somit lange auf die Prävention vorbereitet. Ihre disziplinierte Selbstsorge, ihre verstärkten Leistungsantriebe, ihre forcierte Selbstbezüglichkeit und schließlich, die Appelle an ihre psychische Resilienz gegenüber ihren eigenen Anforderungen erzeugten Subjekte, die für die Burnout-Prävention reif sind.

Im Hinblick auf die Macht-Wissens-Verhältnisse, die das Präventionsdispositiv arrangiert, lässt sich die vorangegangene Argumentation fortführen. Indem die 
Prävention auf das Bändigen der Zukunft ausgerichtet ist, versucht sie, »in der Gegenwart Definitionsmacht über die Zukunft zu gewinnen.« (Kappeler 2016: 62) Wird dann von der »Prävention als tyrannischem Zeitregiment der Gegenwart« (a.a.O.: 57) gesprochen, so impliziert dies im Weiteren, dass sie das Wissen über Zukunft kontinuierlich produzieren, problematisieren und regieren muss. Mit anderen Worten, das Machtregime der Prävention operiert vorausschauend, weil präventiv. Indem sie Probleme herstellt, erscheint sie im Nachhinein als ihre Lösung, als Lösung, die sie selbst vorgesehen hat. Allerdings können Lösungen unterschiedlich ausfallen, je nachdem, welche Version der Zukunft antizipiert wird, womit »zwangsläufig Konflikte verbunden [sind]. Da diese Konflikte ebenfalls durch Macht entschieden werden, setzen sich normalerweise die Präventionsinteressen der Machtzentren durch, so daß Prävention empirisch zur Festigung von gegebenen Machtverhältnissen beiträgt.«(Schülein 1983: 17) Die umgekehrte Seite dieses Bezugs besteht darin, dass das Beherrschen-Wollen von Zeit »regelmäßig sein Gegenteil [produziert], das Beherrscht-Werden von der Zeit« (Kappeler 2016: 62), und zwar nicht nur in dem Sinne, dass die Zukunft genau so kommen muss, wie sie projiziert wurde, sondern auch in dem Sinne, dass die Prävention selbst Zeit erfordert, bis ihre Maßnahmen Wirkung zeigen. Was dann offen bleibt, ist die Frage, wer die Grenzen dafür setzt, wie lange ein konkretes Problem präventiert werden muss, damit es nicht bzw. nicht in dem Maße bedrohlich erscheint, wie dies erwartet wird.

Des Weiteren produziert Prävention immer neue Risiken und parasitiert an ihrer »Sensibilisierung dadurch, dass sie selbst unentwegt Gefahren in Risiken >umoperiert<, deren Vermeidungsmöglichkeit aber andererseits und im selben Zuge mitangezeigt wird, eben als: Prävention.« (Fuchs 2008: 368 [Herv. i. O.]) Die Risiken werden dann an die Körper und Psychen der Subjekte und an ihre diskursive Hervorbringung geknüpft. So können mögliche Risiken schon bei der vorgeburtlichen Untersuchung (engl. Screening) produziert werden, um Anomalien festzustellen (vgl. Hellerich 1983: 138f), oder anhand der Familiendiagnose bestimmt, um Abweichungen zu beobachten (vgl. a.a.O.: 142f). Selbst eine gelebte Andersartigkeit, eine non-konforme Lebensweise kann risikoreich und daher behandlungsbedürftig erscheinen (vgl. a.a.O.: 145f). Damit wird »das Konzept des Risikos zum zentralen Bestandteil, ja, zur wissenschaftlich-technischen Voraussetzung der neuesten Methoden der Früherkennung und Prävention. (Schulz \& Wambach 1983: 8) Risiko fungiert dann durch seine wissensbasierte Differenzierung als Generator neuer Probleme, auf die sich die präventiven Maßnahmen beziehen und so »in das gesamte menschliche Leben eindringen wollen.«(a.a.O.: 10) 